\begin{tabular}{|c|l|}
\hline Title & On a stochastic difference equation for the multidimensional weakly stationary process with discrete time \\
\hline Author(s) & Okabe, Y asunori \\
\hline Citation & Hokkaido University Preprint Series in Mathematics, 13, 1-43 \\
\hline Issue Date & 1987-09 \\
\hline DOI & 10.14943/49133 \\
\hline Doc URL & http://eprints3.math.sci.hokudai.ac.jp $909 /$ http://hdl.handle.net/2115/45531 \\
\hline Type & bulletin (article) \\
\hline File Information & pre13.pdf \\
\hline
\end{tabular}

Instructions for use 
On a stochastic difference equation

for the multi-dimensional weakly

stationary process with discrete time

Yasunori Okabe

Series \#13. September 1987 


\section{HOKKAIDO UNIVERSITY PREPRINT SERIES IN MATHEMATICS}

\# Author Title

1. Y. Okabe, on the theory of discrete KMO-Langevin equations with reflection positivity ( 1 )

2. Y. Giga and T. Kambe, Large time behavior of the vorticity of two-dimensional flow and its application to vortex formation

3. A. Arai, Path Integral Representation of the Index of Kahler-Dirac operators on an Infinite Dimensional Manifold

4. 1. Nakamura, Threefolds Homeomorphic to a Hyperquadric in P4

5. T. Nakazi, Notes on Interpolation by Bounded Analytic Functions

6. T. Nakazi, A Spectral Dilation of Some Non-Dirichlet Algebra

7. H. Hida, A p-adic measure attached to the zeta functions associated with two elliptic modular forms 11

8. T. Suwa, A factorization theorem for unfoldings of analytic functions

9. T. Nakazi, Weighted norm inequalities and uniform algebras

10. T. Miyake, on the spaces of Eisenstein series of Hilbert modular groups

11. K. Nitta, Note on the double centralizers in an H-separable extension

12. K. Kubota, Microlocal parametrices and propagation of singularities near gliding points for hyperbolic mixed problems II 


\title{
On a stochastic difference equation for the multi- \\ dimensional weakly stationary process \\ with discrete time
}

\author{
Dedicated to Professor Mikio Sato \\ on his sixtieth birthday
}

By Yasunori Okabe*)

*) Partially supported by the Grant-in-Aid for Scientific Research of the Ministry of Education, Science and Culture of Japan. 


\section{§1. Introduction}

The researches for the problem of linear prediction of a onedimensional weakly stationary process $X$ can be classified according to the difference whether the length of prediction interval is infinitely long (Kolmogorov [4], Wiener [28] and Dym-McKean [3]) or finitely limited (Krein [5] and Dym-McKean [2]). The method is to reformulate the above problem in terms of a real Hilbert space $L^{2}(\mathbb{R}, \Delta(d \lambda))$ obtained by closing the linear hull of $\left\{e^{i t \cdot} ; t \in \mathbb{R}\right\}$, where $\Delta(d \lambda)$ is the spectral measure of $X$. In particular, besides a traditional spectral theory, Krein has developed the so-called Krein's theory for the inverse spectral problem, and Dym and McKean, Jr. have reconstructed the theory of Hilbert spaces of entire functions developed by de Branges ([1]).

On the other hand, the author has studied the problem of linear prediction for $X$ from the viewpoint of the theory of stochastic differential equations ([10],[12] [27]). The bud of its spirit can be found in [11], where Sato's hyperfunctions play an importart role in the study of infinitely multiple Markovian property. It is important, not only from a point of view of statistical physics, but also from a probabilistic point of view, to derive a stochastic equation of motion describing the time evolution of $X$. In particular, the process $X$ with reflection positivity has been investigated in detail with the project of clarifying a mathematical structure of the so-called fluctuation-dissipation theorem in statistical physics (Kubo [6] and Mori [9]). In the course of these investigations, it has been found that the time evolution of $X$ can be described by two kinds of Langevin equations with a notable difference in character of 
random forces $([18],[19])$ : One is the first KMO-Langevin equation having a white noise as a random force and the other is the second KMO-Langevin equation where a colored noise named the Kubo noise is taken to be a random force. It is a key to obtain the structure theorem of the outer function of $X$. Since the Fourier transform of the outer function gives the canonical representation kernel for $X$, the author's studies are related to the case of long time prediction interval, except [16] treated by an innovation method.

Following the same spirit, Miyoshi ([7] and [8]) has derived and then characterized a stochastic differential equation for multidimensional weakly stationary process, by referring de Branges's theory for a multi-dimensional case and using Krein's method, which is said to be $(\alpha, \beta, \gamma, \delta)$-Langevin equation and treates the case of finite time prediction interval.

Recently, the author ([24],[25] and [26]) derived and then characterized two kinds of stochastic difference equations for onedimensional weakly stationary time series with reflection positivity, by using the result obtained in the continuous-time case, which are called the first and second KMO-Langevin equation, related to the case of long time prediction interval.

The purpose of the present paper is to derive and then characterize a stochastic difference equation for multi-dimensional weakly stationary time series of a general type, by using the innovation method. And we call it $\mathrm{KM}_{2} \mathrm{O}$-Langevin equation and treat the case of finite time prediction interval. The notable point is that a random force in $\mathrm{KM}_{2} \mathrm{O}$-Langevin equation is not always a white noise, different from the one in $(\alpha, \beta, \gamma, \delta)$-Langevin equation. 
We will state the content of this paper. Let $X=(X(n) ; n \in Z)$ be a d-dimensional weakly stationary process with mean vector zero and covariance matrix $R$. We define for each $n \in \mathbb{N}$ a block Toeplitz matrix $S_{n}(E M(n d ; \mathbb{R}))$ by

$(1.1)$

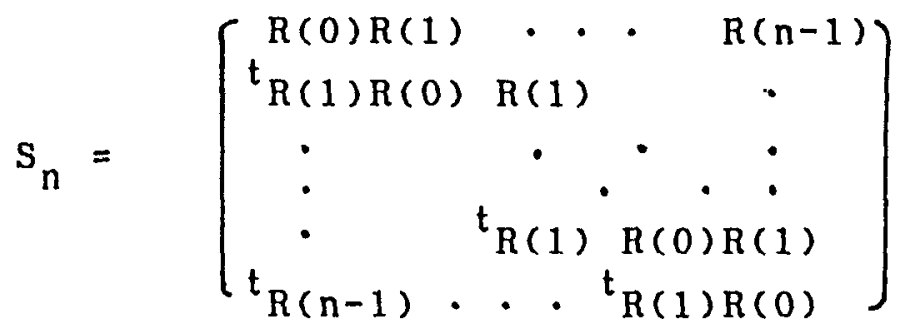

We suppose that $S_{n}(n \in \mathbb{N})$ are invertible in what follows. In $\$ 2$, we will introduce a forward (resp. backward) innovation process I+ $=\left(I_{+}(n) ; n \in \mathbb{N}\right)\left(\right.$ resp. $\left.I_{-}=\left(I_{-}(n) ; n \in \mathbb{N}\right)\right)$ associated with $X$. It is noted that $I_{+}$(resp. I_) is an orthogonal process. And then we derive two kinds of stochastic difference equations describing the time evolution of $x$ :

$$
X(n)=-\sum_{k=1}^{n-1} \gamma_{+}(n, k) X(k)-\delta_{+}(n) X(0)+I_{+}(n) \quad(n \in \mathbb{N})
$$

$$
X(-n)=-\sum_{k=1}^{n-1} \gamma_{-}(n, k) X(-k)-\delta_{-}(n) X(0)+I_{-}(n)(n \in \mathbb{N}) \text {, }
$$

where $\gamma_{+}(\cdot, *), \gamma_{-}(\cdot, *), \delta_{+}(\cdot)$ and $\delta_{-}(\cdot)$ belong to $M(d ; \mathbb{R})$.

We call (1.2)(resp.(1.3)) a forward (resp. backward) $\underline{K M}_{2} \underline{0-}$ Langevin equation for $X$. It is noted that a class of non-linear Langevin equations for strongly stationary time series is derived from our approach (Remark 2.2).

We will in $\$ 3$ obtain fundamental recursive relations among $\gamma_{+}(\cdot, *), \gamma_{-}(\cdot, *), \delta_{+}(\cdot)$ and $\delta_{-}(\cdot)$ (Theorem 3.1): for any $n, k \in$ $\mathbb{N}, \mathrm{n}>\mathrm{k}$,

$$
\begin{array}{ll}
\text { (1.4) } & \gamma_{+}(n, k)=\gamma_{+}(n-1, k-1)+\delta_{+}(n) \gamma_{-}(n-1, n-1-k) \\
(1.5) & \gamma_{-}(n, k)=\gamma_{-}(n-1, k-1)+\delta_{-}(n) \gamma_{+}(n-1, n-1-k),
\end{array}
$$


where $\gamma_{+}(n, 0)=\delta_{+}(n)$ and $\gamma_{-}(n, 0)=\delta_{-}(n)$.

We denote by $v_{+}(n)$ (resp. $\left.v_{-}(n)\right)$ the covariance matrix of $I_{+}(n)$ (resp. I- $\left.(n)\right)(n \in \mathbb{N})$. By using (1.4) and $(1.5)$, we will in $\S 4$ obtain fundamental recursive relations among $V_{+}(\cdot), V_{-}(\cdot), R(0)$, $\delta_{+}(\cdot)$ and $\delta_{-}(\cdot)\left(\right.$ Theorem 4.1) : for any $n \in \mathbb{N}^{*}$,

(1.6) $\quad v_{+}(n+1)=\left(I-\delta_{+}(n+1) \delta_{-}(n+1)\right) v_{+}(n)$

(1.7) $\quad v_{-}(n+1)=\left(I-\delta_{-}(n+1) \delta_{+}(n+1)\right) v_{-}(n)$

(1.8) $\quad v_{+}(n)^{t} \delta_{-}(n+1)=\delta_{+}(n+1) v_{-}(n)$,

where $v_{+}(0)=V_{-}(0)=R(0)$.

By taking advantage of the innovation method, we will in $\$ 5$ give a forward (resp. backward) prediction formula for $X$ (Theorems 5.1 and 5.2$)$. It will be found that prediction matrices and prediction error matrices are determined by $R(0), \delta_{+}(\cdot)$ and $\delta_{-}(\cdot)$. As a converse setting of $\$ 2-\S 5$, we will in $\S 6$ show a reconstruction theorem (Theorem 6.1), which states that for a given system $\left\{V, \delta_{+}(n) ; n \in N\right\}$ in $M(d ; \mathbb{R})$ and a d-dimensional orthogonal process $I_{+}=\left(I_{+}(n) ; n \in \mathbb{N}^{*}\right)$, the recursive relations $(1.4)-(1.8)$ characterize the weakly stationarity property of unique solution $X_{+}$ $=\left(X(n) ; n \in \mathbb{N}^{*}\right)$ of the forward $K M_{2}$ O-Langevin equation (1.2), where $I_{+}(0)=X(0), V=R(0)$ and $I_{+}$becomes a forward innovation process associated with $X_{+}$.

In a forthcoming paper ([27]), we will construct an outer matrix function and then derlve a KMO-Langevin equation for a multidimensional weakly stationary time series. And together with some relations between $K M_{2} \mathrm{O}$-Langevin equation and KMO-Langevin equation, generalized fluctuation-dissipation theorems will be proved based on both Langevin equations. 


\section{\$2. $\mathrm{KM}_{2}$ o-Langevin equations}

Let $X=(X(n) ; n \in Z)$ be an $\mathbb{R}^{d}$-valued weakly stationary time series on a probabllity space $(\Omega, B, P)$ with mean vector zero and covariance matrix R :

$$
\text { (2.1) } \quad R(n)=E\left(X(n)^{t} X(0)\right) \quad(n \in Z) \text {. }
$$

Note that

$$
{ }^{t} R(n)=R(-n)
$$$$
(n \in Z) \text {. }
$$

Let $M$ be the closed subspace of $L^{2}(\Omega, B, P)$ defined by (2.3) $\quad M=$ the closed linear hull of $\left(X_{j}(n) ; 1 \leq j \leq d, n \in Z\right)$. We then have the unitary group ( $U(m) ; m \in Z$ ) acting on $M$ such that $(2.4)$

$$
U(m)(X(n))=X(n+m) \quad(m, n \in Z) \text {. }
$$

For each $n \in \mathbb{N}$ we define a block Toeplitz matrix $S_{n} \in$ $M(n d ; \mathbb{R})$ by

$$
S_{n}=\left(\begin{array}{ccccc}
R(0) R(1) & \cdot & \cdot & R(n-1) \\
t_{R(1) R(0)} & R(1) & & \cdot \\
\cdot & \cdot & \cdot & \cdot & \cdot \\
\cdot & & t_{R(1)} & \cdot & \cdot \\
t_{R(n-1)} & \cdot & \cdot & \cdot & t_{R(1)} R(0)
\end{array}\right)
$$

Since

$$
S_{n}=E\left(Y_{n}{ }^{t} Y_{n}\right) \text {, }
$$

where $Y_{n}={ }^{t}\left(X_{1}(n-1), \ldots, X_{d}(n-1), \ldots, X_{1}(0), \cdots, X_{d}(0)\right)$,

we can see that either of the following (2.7) and (2.8) holds:

$$
S_{n} \in G L(n d ; \mathbb{R}) \quad \text { for any } n \in \mathbb{N}
$$

(2.8) there exists $n_{0} \in \mathbb{N}$ such that $s_{n} \in G L(n d ; R)$ for any $n \in\left\{1, \cdots, n_{0}\right\}$ and $s_{n} \notin G L(n d ; \mathbb{R})$ for any $n \in\left(n_{0}+1, n_{0}+2, \cdots\right\}$. We treat the case where condition (2.7) holds in what follows.

Remark 2.1. If $R$ has a spectral density matrix $\Delta=\Delta(\theta)$ 
$(\theta \in[-\pi, \pi))$ such that $\Delta(\theta) \in G L(d ; \mathbb{C})$ for almostall $\theta \in[-\pi, \pi)$, it can be seen that condition (2.7) holds.

For each $n \in \mathbb{N}$ we define the following block Toeplitz matrix $T_{n} \in M(n d ; \mathbb{R})$ by

$(2.9)$

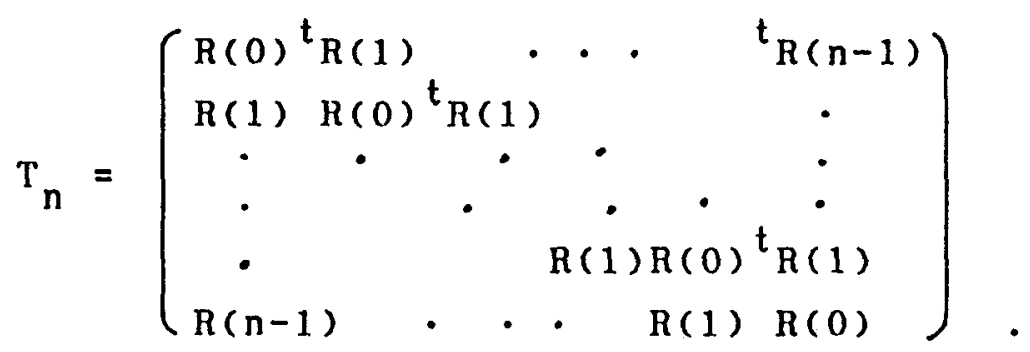

Since

(2.10) $\quad T_{n}=E\left(Z_{n}{ }^{t} Z_{n}\right)$,

where $z_{n}={ }^{t}\left(x_{1}(0), \cdots, x_{d}(0), \cdots, x_{1}(n-1), \cdots, x_{d}(n-1)\right)$,

it holds from (2.6) and (2.7) that

(2.11) $\quad T_{n} \in G L(n d ; \mathbb{R})$ for any $n \in \mathbb{N}$.

For each $n \in \mathbb{N}^{*}=(0,1,2, \cdots)$ we define two closed linear

subspaces $M_{0}^{+}(n)$ and $M_{0}^{-}(n)$ of $M$ by

(2.12) $\quad M_{0}^{+}(n)=$ the linear hull of $\left\{X_{j}(m) ; 1 \leq j \leq d, 0 \leq m \leq n\right\}$

(2.13) $\quad M_{0}^{-}(n)=$ the linear hull of $\left\{X_{j}(-m) ; 1 \leq j \leq d, 0 \leq m \leq n\right)$.

Then we introduce two $\mathbb{R}^{d}$-valued stochastic processes $I_{+}=\left(I_{+}(n)\right.$;

$n \in \mathbb{N})$ and $I_{-}=\left(I_{-}(n) ; n \in \mathbb{N}\right)$ on $(\Omega, Q, P)$ by

$(2.14)$

$(2.15)$

$$
\begin{aligned}
& I_{+}(n)=X(n)-P M_{0}^{+}(n-1) X(n) \\
& I_{-}(n)=X(-n)-P M_{0}^{-}(n-1)
\end{aligned}
$$

where $P_{M_{0}^{+}(n-1)}$ (resp. $P_{M_{0}^{-}(n-1)}$ ) stands for the orthogonal projec-

tion on $M_{0}^{+}(n-1) \quad\left(\operatorname{resp} \cdot M_{0}^{-}(n-1)\right)$.

It follows from condition (2.7) that there uniquely exist two sys tems $\left(\gamma_{+}(n, k), \delta_{+}(m) ; k, m, n \in \mathbb{N}, n>k\right)$ and $\left(\gamma_{-}(n, k), \delta_{-}(m) ; k, m, n \in \mathbb{N}, n>k\right)$ of members in $M(d ; \mathbb{R})$ such that for any $n \in \mathbb{N}$, 
$\begin{aligned} & \text { (2.16) } X(n)=-\sum_{k=1}^{n-1} \gamma_{+}(n, k) X(k)-\delta_{+}(n) X(0)+I_{+}(n) \\ & \text { (2.17) } \quad X(-n)=-\sum_{k=1}^{n-1} \gamma_{-}(n, k) X(-k)-\delta_{-}(n) X(0)+I_{-}(n) .\end{aligned}$

In particular, it holds that for any $n \in \mathbb{N}$,

(2.18) $\quad\left(X(0), I_{+}(m) ; m \in N\right\}$ is orthogonal in $M^{d}$

(2.19) $\quad\left(X(0), I_{-}(m) ; m \in N\right)$ is orthogonal in $M^{d}$

(2.20) $\quad M_{0}^{+}(n)=$ the linear hull of $\left(X_{j}(0), I+j(l) ; 1 \leq j \leq d, 1 \leq l \leq n\right)$

(2.21) $\quad M_{0}^{-}(n)=$ the linear hull of $\left(X_{j}(0), I_{-j}(l) ; 1 \leq j \leq d, 1 \leq l \leq n\right)$.

where $I_{+}(l)={ }^{t}\left(I_{+1}(l), \cdots, I_{+d}(l)\right)$ and $I_{-}(l)={ }^{t}\left(I_{-1}(l), \cdots, I_{-d}(l)\right)$.

We call $I_{+}(r e s p$. I_) a forward (resp. backward) innovation process associated with $X$. Furthermore, by regarding (2.16) (resp.

(2.17)) as a stochastic difference equation describing the time evolution of $x$, we call (2.16) (resp.(2.17)) a forward (resp. backward) $\mathrm{KM}_{2}$ o-Langevin equation for $\mathrm{X}$.

Remark 2.2. Let $Y=(Y(n) ; n \in Z)$ be a one-dimensional strongly stationary time series on $(\Omega, B, P)$ such that

(2.22) $\quad \mathrm{Y}(0) \in L^{4}(\Omega, B, P)$

(2.23) $\quad E(Y(0))=0$.

We define three weakly stationary time series $x^{(j)}=\left(X^{(j)}(n) ; n \in Z\right)$

$(j=1,2,3) \quad$ by

$(2.24)$

$$
X^{(1)}(n)=\left(\begin{array}{l}
Y(n) \\
Y(n)^{2}-E\left(Y(0)^{2}\right)
\end{array}\right)
$$

$(2.25)$

(2.26)

$$
X^{(2)}(n)=\left(\begin{array}{l}
Y(n) \\
Y(n) Y(n-1)-E(Y(1) Y(0))
\end{array}\right)
$$

$$
X^{(3)}(n)=\left(\begin{array}{l}
Y(n) \\
Y(n)^{2}-E\left(Y(0)^{2}\right) \\
Y(n) Y(n-1)-E(Y(1) Y(0))
\end{array}\right) \text {. }
$$


If we suppose condition (2.7) for $x^{(j)}(j=1,2,3)$, we can derive three non-linear Langevin equations for $Y$ through $\mathrm{KM}_{2} \mathrm{O}$-Langevin equations for $X^{(j)}(j=1,2,3)$. The problem of non-linear prediction for $Y$ will be investigated based on these non-linear Langevin equations in the near future. 
§3. Relations among $\gamma_{+}(\cdot, *), \gamma_{-}(\cdot, *), \delta_{+}(\cdot)$ and $\delta_{-}(\cdot)$

For convenience sake, we set for each $n \in \mathbb{N}$

$$
\begin{aligned}
& \gamma_{+}(n, 0)=\delta_{+}(n) \\
& \gamma_{-}(n, 0)=\delta_{-}(n) .
\end{aligned}
$$

Theorem 3.1. For any $n, k \in \mathbb{N}, n>k$,

$$
\begin{aligned}
& \gamma_{+}(n, k)=\gamma_{+}(n-1, k-1)+\delta_{+}(n) \gamma_{-}(n-1, n-1-k) \\
& \gamma_{-}(n, k)=\gamma_{-}(n-1, k-1)+\delta_{-}(n) \gamma_{+}(n-1, n-1-k) .
\end{aligned}
$$

Proof. Fix any $n \in(2,3, \cdots)$. By multiplying both hand sides of equation (2.16) by ${ }^{t} X(m)(m=0,1, \cdots, n-1)$ and then taking an expectation with respect to the probability $P$, we see from (2.18) and (2.20) that for any $m \in(0,1, \cdots, n-1)$,

$$
(3.3)_{m} \quad R(n-m)=-\sum_{k=1}^{n-1} \gamma_{+}(n, k) R(k-m)-\delta_{+}(n){ }^{t} R(m) \text {. }
$$

By replacing $n$ in $(3.3)_{m}$ by $n-1$, we have, for any $m \in(0,1$, $\cdots, n-2)$,

$$
\text { (3.4) } \mathrm{m} \quad \mathrm{R}(\mathrm{n}-1-\mathrm{m})=-\sum_{\mathrm{k}=0}^{\mathrm{n}-2} \gamma_{+}(n-1, k) R(k-m) \text {. }
$$

On the other hand, by multiplying both hand sides of equation ( 2 . 17) replaced $n$ by $n-1$ by ${ }^{t} x(-m)(m=0,1, \cdots, n-2)$ and then taking an expectation with respect to $P$, we see from (2.19) and (2.21) that for any $m \in\{0,1, \cdots, n-2\}$,

$$
\text { (3.5 })_{m} \quad t_{R(n-1-m)}=-\sum_{k=0}^{n-2} \gamma_{-}(n-1, k){ }^{t} R(k-m) \text {. }
$$

Now, let any $m \in(1,2, \cdots, n-1)$ be fixed. By combining $R(n-m)$ (resp. $t_{R(m))}$ in $(3.3)_{m}$ with $R(n-m)$ in $(3.4)_{m-1}$ (resp. $t_{R(m)}$ in $\left.(3.5)_{n-1-m}\right)$, we have

$$
-\sum_{k=0}^{n-2} \gamma_{+}(n-1, k) R(k+1-m)
$$




$$
\begin{aligned}
& =-\sum_{k=1}^{n-1} \gamma_{+}(n, k) R(k-m)+\delta_{+}(n) \sum_{k=0}^{n-2} \gamma_{-}(n-1, k){ }^{t} R(k-n+1+m) \\
& =-\sum_{k=0}^{n-2}\left(\gamma_{+}(n, k+1)-\delta_{+}(n) \gamma_{-}(n-1, n-2-k)\right) R(k+1-m)
\end{aligned}
$$

and so

$$
\sum_{k=0}^{n-2}\left(\gamma_{+}(n, k+1)-\gamma_{+}(n-1, k)-\delta_{+}(n) \gamma_{-}(n-1, n-2-k)\right) R(k+1-m)=0 .
$$

Therefore, it follows from condition (2.7) that (i) holds. Similarly, we can prove (ii). (Q.E.D.)

For future use in $\$ 6$, for any $m \in \mathbb{N}^{*}$ and $n \in \mathbb{N}$, we set (3.6)

$$
\begin{aligned}
& n_{+}(m, n)=\gamma_{+}(m+n, m) \\
& n_{-}(m, n)=\gamma_{-}(m+n, m) .
\end{aligned}
$$

Immediately from Theorem 3.1 , we have

Theorem 3.1. For any $m \in \mathbb{N}^{*}$ and $n \in \mathbb{N}$,

$$
\begin{aligned}
& n_{+}(m+1, n)=n_{+}(m, n)+\delta_{+}(m+n+1) n_{-}(n-1, m+1) \\
& n_{-}(m+1, n)=n_{-}(m, n)+\delta_{-}(m+n+1) n_{+}(n-1, m+1) .
\end{aligned}
$$


§4. Relations among $v_{+}(\cdot), v_{-}(\cdot), \delta_{+}(\cdot)$ and $\delta_{-}(\cdot)$

For convenience sake, we put

(4.1)

$$
I_{+}(0)=I_{-}(0)=X(0)
$$

and denote by $v_{+}(n)$ (resp. $v_{-}(n)$ ) the covariance matrix of $I_{+}(n)$ (resp. I_ $(n))\left(n \in \mathbb{N}^{*}\right)$ :

$$
v_{+}(n)=E\left(I_{+}(n)^{t} I_{+}(n)\right)
$$

$$
v_{-}(n)=E\left(I_{-}(n)^{t} I_{-}(n)\right) \text {. }
$$

Lemma 4.1 (i) det $S_{n}=\prod_{k=0}^{n-1}$ det $v_{+}(k)$ for any $n \in \mathbb{N}$

$$
\begin{aligned}
& \text { det } T_{n}=\prod_{k=0}^{n-1} \text { det } v_{-}(k) \\
& v_{+}(n), v_{-}(n) \in G L(n d ; \mathbb{R})
\end{aligned}
$$

for any $n \in \mathbb{N}$ for any $n \in \mathbb{N}$.

Proof. (i) for $n=1$ is trivial. Let any $n \in(2,3, \ldots\}$ be fixed. By multiplying both hand sides of equation (2.16) by ${ }^{t} X(n)$ and then taking an expectation with respect to $P$, we see from (2.18) and $(2.20)$ that

$$
R(0)=-\sum_{k=1}^{n-1} \gamma_{+}(n, k)^{t} R(n-k)-\delta_{+}(n){ }^{t} R(n)+v_{+}(n) .
$$

By making a matrix representation of (4.4) and $(3.3)_{m}(m=n-1, n-2$, $\cdots, 1,0)$, we have

$$
\left(\begin{array}{cccc}
I \gamma_{+}(n, n-1) & \cdots \gamma_{+}(n, 1) \delta_{+}(n) \\
I & 0 & \\
& & & \\
0 & & &
\end{array}\right) S_{n+1}=\left(\begin{array}{cc}
v_{+}(n) & 0 \cdots 0 \\
t_{R(1)} & \\
\vdots & s_{n} \\
t_{R(n)} &
\end{array}\right)
$$

which yields (i). (ii) is also proved similarly. (iii) follows from (2.7), (2.11), (i) and (i i). (Q.E.D.)

Lemma 4.2 . For any $n \in \mathbb{N}^{*}$, 


$$
\begin{aligned}
& R(n+1)=-\delta_{+}(n+1) v_{-}(n)-\sum_{k=0}^{n-1} \gamma_{+}(n, k) R(k+1) \\
& t_{R(n+1)}=-\delta_{-}(n+1) v_{+}(n)-\sum_{k=0}^{n-1} \gamma_{-}(n, k){ }^{t} R(k+1) .
\end{aligned}
$$

Proof. Since $v_{+}(n)$ is a symmetric matrix, it follows from (4.4) that for any $n \in \mathbb{N}^{*}$,

$$
v_{+}(n)=R(0)+\sum_{k=0}^{n-1} R(n-k)^{t} \gamma_{+}(n, k)
$$

Similarly,

$$
v_{-}(n)={ }^{t} R(0)+\sum_{k=0}^{n-1} t h(n-k)^{t} \gamma_{-}(n, k) .
$$

By replacing $n$ by $n+1$ in (3.3) 0 and then using Theorem $3.1(i)$,

$$
R(n+1)=-\delta_{+}(n+1)\left(\sum_{k=0}^{n-1} \gamma_{-}(n, k) R(n-k)+R(0)\right)-\sum_{k=0}^{n-1} \gamma_{+}(n, k) R(k+1) \text {, }
$$

which, together with (4.6), Implies (1). (ii) is also proved similar$1 \mathrm{y}$.

(Q.E.D.)

Now, we will show the following fundamental

Lemma 4.3. For any $n \in \mathbb{N}$.

$$
\sum_{k=0}^{n-1} n_{+}(k, n-k) R(k+1)=\sum_{k=0}^{n-1} R(k+1)^{t} n_{-}(k, n-k) \text {. }
$$

The proof is divided into 13 steps. We denote by $A_{n}$ the right hand side minus the left hand side in Lemma 4.3 :

$$
A_{n}=\sum_{k=0}^{n-1} R(k+1)^{t} n_{-}(k, n-k)-\sum_{k=0}^{n-1} n_{+}(k, n-k) R(k+1) \text {. }
$$

(step 1) (i) $v_{+}(n+1)=v_{+}(n)-\delta_{+}(n+1) v_{-}(n)^{t} \delta_{+}(n+1)+A_{n}$

$$
v_{-}(n+1)=v_{-}(n)-\delta_{-}(n+1) v_{+}(n){ }^{t} \delta_{-}(n+1)-{ }^{t} A_{n}
$$

$$
v_{+}(n){ }^{t} \delta_{-}(n+1)-\delta_{+}(n+1) v_{-}(n)=A_{n} \text {. }
$$

Proof. By applying Theorem 3.1 to (4.5),

$$
v_{+}(n+1)=v_{+}(n)+\left(\sum_{k=0}^{n-1} R(k+1)^{t} n_{-}(k, n-k)+R(n+1)\right)^{t} \delta_{+}(n+1) \text {. }
$$


which, together with Lemma 4.2 (i), implies (i). (ii) is also proved similarly. By using Lemma 4.2 again, we get (iii).

(step 2) (i) $\mathrm{R}(1)=-\delta_{+}(1) \mathrm{R}(0)$

(i i ) $\quad t_{R(1)}=-\delta_{-}(1) R(0)$

(i i i ) $\quad R(0)^{t} \delta_{-}(1)=\delta_{+}(1) R(0)$

(iv) $\quad v_{+}(1)=\left(I-\delta_{+}(1) \delta_{-}(1)\right) R(0)$

(v) $\quad v_{-}(1)=\left(I-\delta_{-}(1) \delta_{+}(1)\right) R(0)$.

Proof. By multiplying both hand sides of (2.16) and (2.17) for $n=1$ by ${ }^{t} x(0)$ and then taking an expectation with respect

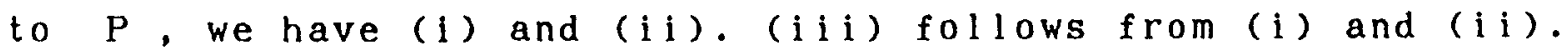
Next, by multiplying (2.16) for $n=1$ by ${ }^{t} x(1)$ and then taking an expectation, we see from $(2.18),(2.20),(1)$ and (i i) in Step 2 that (iv) holds.(v) is also proved similarly. (Q.E.D.) (Step 3) $\quad A_{1}=0$.

This follows from (iil) in step 2 . (Step 4) For any $n \in(2,3, \cdots)$,

$$
A_{n}=\delta_{+}(n) I_{n}^{(1) t} \delta_{-}(n)+\delta_{+}(n) I_{n}^{(1)}+\mathbb{I I I}_{n}^{(1) t} \delta_{-}(n)+I V_{n}^{(1)} \text {, }
$$

where

$$
\begin{aligned}
I_{n}^{(1)}= & v_{-}(n-1)^{t} \delta_{+}(n-1)-\delta_{-}(n-1) v_{+}(n-1) \\
I_{n}^{(1)}= & -\left(R(1)+\sum_{j=1}^{n-2} n_{-}(n-1-j, j) R(j+1)\right)+ \\
& +\delta_{-}(n-1) \sum_{j=0}^{n-2} R(j+1)^{t} n_{-}(j, n-1-j)-v_{-}(n-1)^{t} n_{-}(n-2,1) \\
I_{n}^{(1)}= & R(1)+\sum_{j=1}^{n-2} R(j+1)^{t} n_{+}(n-1-j, j)- \\
& -\left(\sum_{j=0}^{n-2} n_{+}(j, n-1-j) R(j+1)\right)^{t} \delta_{+}(n-1)+n_{+}(n-2,1) v_{+}(n-1) \\
I V_{n}^{(1)=} & \sum_{j=0}^{n-3} n_{+}(j, n-1-j) R(j+2)+\left(\sum_{j=0}^{n-3} n_{+}(j, n-1-j) R(j+1)\right)^{t} n_{-}(n-2,1)-
\end{aligned}
$$




$$
-\sum_{j=0}^{n-3} R(j+2)^{t} n_{-}(j, n-1-j)-n_{+}(n-2,1) \sum_{j=0}^{n-3} R(j+1)^{t} n_{-}(j, n-1-j) \text {. }
$$

This decomposition follows from Theorem $3.1^{\prime}$ and Lemma 4.2 .

(Step 5) For any $n \in\{2,3, \cdots\}$ and any $k \in\{1,2, \cdots, n-1\}$,

$$
\begin{aligned}
I V_{n}^{(k)}= & \delta_{+}(n-k) I_{n}^{(k+1) t} \delta_{-}(n-k)+\delta_{+}(n-k) I_{n}^{(k+1)}+ \\
& +\mathbb{I I}_{n}^{(k+1) t} \delta_{-}(n-k)+I V_{n}^{(k+1)},
\end{aligned}
$$

where

$$
\begin{aligned}
& I_{n}^{(k+1)}=-\sum_{j=0}^{k-1} n_{-}(j, n-k-1-j)\left(R(k-j)+\sum_{i=1}^{n-2 k-1} R(k-j+i)^{t} n_{+}(n-k-1-i, i)\right)+ \\
& +\sum_{j=0}^{k-1}\left(R(k-j)+\sum_{i=1}^{n-2 k-1} n_{-}(n-k-1-i, i) R(k-j+1)\right) n_{+}(j, n-k-j)- \\
& -n_{-}(k, n-2 k-1) v_{+}(n-k-1)+v_{-}(n-k-1)^{t} n_{+}(k, n-2 k-1) \\
& \Pi_{n}^{(k+1)}=R(k+1)+\sum_{j=1}^{n-2 k-2} n_{-}(n-k-1-j, j) R(k+1+j)- \\
& -\sum_{j=0}^{k} n_{-}(j, n-k-1-j)\left(\sum_{i=0}^{n-2 k-2} R(k-j+1+i)^{t} n_{-}(i, n-k-1-i)\right)+ \\
& +\sum_{j=1}^{k}\left(R(k+1-j)+\sum_{i=1}^{n-2 k-2} n_{-}(n-k-1-i, i) R(k+1-j+i)\right)^{t} n_{-}(n-k-1-j, j)+ \\
& +v_{-}(n-k-1)^{t} n_{-}(n-2 k-2, k+1) \\
& \text { III }_{n}^{(k+1)}=-\left(R(k+1)+\sum_{j=1}^{n-2 k-2} R(k+1+j) t n_{+}(n-k-1-j, j)\right)+ \\
& +\sum_{j=0}^{k}\left(\sum_{i=0}^{n-2 k-2}{ }^{t} n_{+}(i, n-k-1-i) R(k-j+1+i)\right)^{t} n_{+}(j, n-k-1-j)- \\
& -\sum_{j=1}^{k} n_{+}(n-k-1-j, j)\left(R(k+1-j)+\sum_{i=1}^{n-2 k-2} R(k+1-j+i) n_{+}(n-k-1-i, i)\right)- \\
& -n_{+}(n-2 k-2, k+1) v_{+}(n-k-1) \\
& I V_{n}^{(k+1)}=\sum_{j=0}^{n-2 k-3} n_{+}(j, n-k-1-j) R(k+2+j)-\sum_{j=0}^{n-2 k-3} R(k+2+j){ }^{t} n_{-}(j, n-k-1-j)- \\
& -\sum_{j=1}^{k+1} n_{+}(n-k-1-j, j)\left(\sum_{i=0}^{n-2 k-3} R(k+1+i)^{t} n_{-}(i, n-k-1-i)\right)+ \\
& +\sum_{j=1}^{k+1}\left(\sum_{i=0}^{n-2 k-3} n_{+}(i, n-k-1-i) R(k+1+i)\right)^{t} n_{-}(n-k-1-j, j) \text {. }
\end{aligned}
$$

Similarly to step 4, this follows from Theorem 3.1 ' and Lemma 4.2 . 
(Step 6) (1) For any even $n=2 N(N \in N), I V_{n}^{(N)}=0$.

(ii) For any odd $n=2 N+1$ (NEN),

$$
\begin{aligned}
I V_{n}^{(N)}=\delta_{+}(N+1) & 1-V_{+}(N)-\sum_{j=0}^{N-1} v_{+}(N-1-j)^{t} \delta_{-}(N-j)^{t} \delta_{+}(N-j)+ \\
& \left.+V_{-}(N)+\sum_{j=0}^{N-1} \delta_{-}(N-j) \delta_{+}(N-j) v_{-}(N-1-j)\right\}^{t} \delta_{-}(N+1) .
\end{aligned}
$$

Proof. Immediately from Step 5, we have (i). (ii) can be proved as follows: By Step 5,

$$
\begin{aligned}
I V_{n}^{(N)}= & \delta_{+}(N+1)\left(R(N+1)+\sum_{j=1}^{N} R(N+1-j)^{t} n_{-}(N+1-j, j)\right)- \\
& -\left(R(N+1)+\sum_{j=1}^{N} n_{+}(N+1-j, j) R(N+1-j)\right)^{t} \delta_{-}(N+1) .
\end{aligned}
$$

By Theorem 3.1' and Lemma 4.2,

$$
\begin{aligned}
& R(N+1)+\sum_{j=1}^{N} R(N+1-j)^{t} n_{-}(N+1-j, j)=\left(-V_{+}(N)+\sum_{j=0}^{N-1} R(N-j)^{t} n_{+}(j, N-j)\right)^{t} \delta_{-}(N+1) \\
& R(N+1)+\sum_{j=1}^{N} n_{+}(N+1-j, j) R(N+1-j)=\delta_{+}(N+1)\left(-V_{-}(N)+\sum_{j=0}^{N-1} n_{-}(j, N-j) R(N-j)\right) .
\end{aligned}
$$

And so

$$
\begin{aligned}
I V_{n}^{(N)}=\delta_{+}(N+1)\left(-V_{+}(N)\right. & +\sum_{j=0}^{N-1} R(N-j)^{t} n_{+}(j, N-j)- \\
& \left.-V_{-}(N)+\sum_{j=0}^{N-1} n_{-}(j, N-j) R(N-j)\right)^{t} \delta_{-}(N+1)
\end{aligned}
$$

Furthermore, by using Theorem 3.1' and Lemma 4.2 again,

$$
\begin{aligned}
& \sum_{j=0}^{N-1} R(N-j)^{t} n_{+}(j, N-j)=-v_{+}(N-1)^{t} \delta_{-}(N)^{t} \delta_{+}(N)+\sum_{j=0}^{N-2} R(N-1-j)^{t} n_{+}(j, N-1-j) \\
& \sum_{j=0}^{N-1} n_{-}(j, N-j) R(N-j)=-\delta_{-}(N) \delta_{+}(N) v_{-}(N-1)+\sum_{j=0}^{N-2} n_{-}(j, N-1-j) R(N-1-j),
\end{aligned}
$$

which yields

$$
\begin{aligned}
& I V_{n}^{(N)}=\delta_{+}(N+1)\left(-V_{+}(N)-V_{+}(N-1)^{t} \delta_{-}(N)^{t} \delta_{+}(N)+\right. \\
& +\sum_{\substack{j=0 \\
N-2}}^{N-2} R(N-1-j)^{t} n_{+}(j, N-1-j)+V_{-}(N)+\delta_{-}(N) \delta_{+}(N) V_{-}(N-1)- \\
& \left.-\sum_{j=0}^{N-2} n_{-}(j, N-1-j) R(N-1-j)\right\}^{t} \delta_{-}(N+1) \text {. }
\end{aligned}
$$


By repeating the same procedure, we see from step 2 that (i) holds. (ii) follows immediately from step 5 . (Q.E.D.)

(Step 7) For any fixed $n_{0} \in \mathbb{N}^{*}$, if $v_{+}\left(n_{0}\right)^{t} \delta_{-}\left(n_{0}+1\right)=$ $\delta_{+}\left(n_{0}+1\right) v_{-}\left(n_{0}\right)$, then $v_{-}\left(n_{0}+1\right)^{t} \delta_{+}\left(n_{0}+1\right)=\delta_{-}\left(n_{0}+1\right) v_{+}\left(n_{0}+1\right)$.

Proof. By Step 1, (ii), (iv) and (v) in Step 2,

$$
\begin{aligned}
v_{-}\left(n_{0}+1\right){ }^{t} \delta_{+}\left(n_{0}+1\right) & =\left(I-\delta_{-}\left(n_{0}+1\right) \delta_{+}\left(n_{0}+1\right)\right) v_{-}\left(n_{0}\right){ }^{t} \delta_{+}\left(n_{0}+1\right) \\
& =\delta_{-}\left(n_{0}+1\right)\left(1-\delta_{+}\left(n_{0}+1\right) \delta_{-}\left(n_{0}+1\right)\right) v_{+}\left(n_{0}\right) \\
& =\delta_{-}\left(n_{0}+1\right) v_{+}\left(n_{0}+1\right) .
\end{aligned}
$$

(Step 8) $I_{n}^{(1)}=I_{n}^{(1)}=I_{n}^{(1)}=I V_{n}^{(1)}=0$ for any $n \in(2,3)$.

Proof. By (i i i) in Step 2 (resp.Step 3) with step 7 , we have $I_{2}^{(1)}=0\left(\operatorname{resp} \cdot I_{3}^{(1)}=0\right) \cdot \mathbb{I}_{2}^{(1)}$ and $\mathbb{I}_{2}^{(1)}$ follow from step 2 . A simple calculation, together with Lemma 4.2 and $\mathrm{Step} 2$, yields II (1) and $\mathrm{III}_{3}^{(1)} \cdot \mathrm{IV}_{\mathrm{n}}^{(1)}(n=2,3)$ follow from Steps 2 and $6 . \quad$ (Q.E.D.)

Now, by a mathematical induction, we shall show the following statement $(*)_{n}(n=2,3, \cdots):$ for any $m \in\{2,3, \cdots, n\}$,

$$
(*)_{n} \begin{cases}(i) \quad I_{m}^{(k)}=\mathbb{I}_{m}^{(k)}=\mathbb{I}_{m}^{(k)}=0 \quad(1 \leq k \leq M) \text { if } m=2 M+1 \\ I V_{m}^{(M)}=0 \\ (i i) \quad I_{m}^{(k)}=\mathbb{I}_{m}^{(k)}=\mathbb{I}_{m}^{(k)}=0 \quad(1 \leq k \leq M) \text { if } m=2 M .\end{cases}
$$

Step 8 implies that $(*){ }_{n}(n=2,3)$ hold. For any fixed $n_{0} \in(2,3$, $\cdots$, , let us suppose that $(*)_{n_{0}}$ holds. Moreover we consider the case where $n_{0}$ is even, $n_{0}=2 N_{0}$. The case where $n_{0}$ is odd is proved similarly. What we have to show is that

$$
\begin{array}{ll}
(*)_{n_{0}, k}^{\prime} & I \frac{(k)}{n_{0}+1}=\Pi_{n_{0}+1}^{(k)}=I_{n_{0}+1}^{(k)}=0 \quad\left(1 \leq k \leq N_{0}\right), \\
(*)_{n_{0}, N_{0}}^{\left(N_{0}\right)} & I V_{n_{0}+1}^{(n)}=0 .
\end{array}
$$


Since $\quad(*){ }_{n_{0}}$ implies that $A_{m}\left(2 \leq m \leq n_{0}\right)$ hold, it follows from Steps 1

and 7 that for any $m \in\left(2, \cdots, n_{0}\right)$,

$(4.8)$

$v_{+}(m+1)=\left(I-\delta_{+}(m+1) \delta_{-}(m+1)\right) v_{+}(m)$

(4.9)

$v_{-}(m+1)=\left(I-\delta_{-}(m+1) \delta_{+}(m+1)\right) v_{-}(m)$

$(4.10)$

$v_{+}(m)^{t} \delta_{-}(m+1)=\delta_{+}(m+1) v_{-}(m)$

$(4.11)$

$$
v_{+}(m+1)^{t} \delta_{-}(m+1)=\delta_{+}(m+1) v_{-}(m+1) \text {. }
$$

(

$$
I_{n_{0}+1}^{(1)}=0 \text {. }
$$

This follows from Step 4 and (4.11).

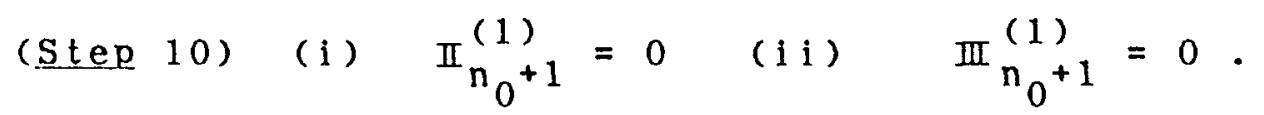

Proof. We prove only (i), since (i i) is shown similarly. By Theorem $3.1^{\prime}$, Lemma $4.2(\mathrm{i} i)$, and the assumption $\mathbb{I}_{\mathrm{n}_{0}}^{(1)}=0$

$$
\begin{aligned}
& \text { III } \begin{array}{l}
(1) \\
n_{0}+1
\end{array} \\
& =-v_{-}\left(n_{0}-1\right)^{t} n_{-}\left(n_{0}-2,1\right)-\delta_{-}\left(n_{0}-1\right) v_{+}\left(n_{0}-1\right)^{t} \delta_{-}\left(n_{0}\right)+v_{-}\left(n_{0}\right)^{t} n_{-}\left(n_{0}-1,1\right)+ \\
& \quad+\delta_{+}\left(n_{0}\right)\left(\sum_{j=0}^{n_{0}-2} n_{+}\left(j, n_{0}-1-j\right) R(2+j)-\sum_{j=0} R(1+j)^{t} n_{-}\left(j, n_{0}-j\right)\right) .
\end{aligned}
$$

Since

$$
v_{-}\left(n_{0}-1\right)^{t} n_{-}\left(n_{0}-2,1\right)+\delta_{-}\left(n_{0}-1\right) v_{+}\left(n_{0}-1\right)^{t} \delta_{-}\left(n_{0}\right)=v_{-}\left(n_{0}-1\right)^{t} n_{-}\left(n_{0}-1,1\right),
$$

we apply Theorem $3.1^{\prime}$ again to find that

$$
\begin{aligned}
\Pi_{n_{0}+1}^{(1)}=\left(v_{-}\left(n_{0}\right)\right. & \left.-v_{-}\left(n_{0}-1\right)\right)^{t} n_{-}\left(n_{0}-1,1\right)+ \\
+ & \delta_{-}\left(n_{0}\right)\left(\sum_{j=0}^{n_{0}-3} n_{+}\left(j, n_{0}-1-j\right) R(2+j)-\sum_{j=0} R(2+j) t n_{-}\left(j, n_{0}-1-j\right)+\right. \\
& +n_{+}\left(n_{0}-2,1\right) R\left(n_{0}\right)-R\left(n_{0}\right)^{t} n_{-}\left(n_{0}-2,1\right)- \\
& \left.-\left(R(1)+\sum_{0}^{-2} \sum_{j=0} R\left(n_{0}-j\right)^{t} n_{+}\left(j, n_{0}-1-j\right)\right)^{t} \delta_{-}\left(n_{0}\right)\right) .
\end{aligned}
$$

By using $I_{n_{0}}^{(1)}=0$, coming from $(*) n_{0}$ and step 5 , we see that

$$
\text { III } n_{0}+1=\left(V_{-}\left(n_{0}\right)-v_{-}\left(n_{0}-1\right)\right)^{t} n_{-}\left(n_{0}-1,1\right)+
$$




$$
\begin{aligned}
& +\delta_{-}\left(n_{0}\right)\left\{n_{+}\left(n_{0}-2,1\right)\left(\sum_{j=0}^{n_{0}-3} R(1+j)^{t} n_{-}\left(j, n_{0}-1-j\right)+R\left(n_{0}\right)\right)-\right. \\
& n_{0}-3 \\
& -\left(\sum_{j=0} n_{+}\left(j, n_{0}-1-j\right) R(1+j)+R\left(n_{0}\right)\right)- \\
& \left.-\left(R(1)+\sum_{j=0}^{n_{0}-2} R\left(n_{0}-j\right)^{t} n_{+}\left(j, n_{0}-1-j\right)\right)^{t} \delta_{-}\left(n_{0}\right)\right) .
\end{aligned}
$$

By using Lemma $4.2(1)(i 1)$ and $m_{n_{0}}^{(1)}=0$,

$$
\begin{aligned}
\pi_{n_{0}+1}^{(1)} \\
=\left(v_{-}\left(n_{0}\right)-v_{-}\left(n_{0}-1\right)\right)^{t} n_{-}\left(n_{0}-1,1\right)+\delta_{-}\left(n_{0}\right) \delta_{+}\left(n_{0}\right) v_{-}\left(n_{0}-1\right)^{t} n_{-}\left(n_{0}-2,1\right)- \\
-\delta_{0}\left(n_{0}\right)\left(n_{+}\left(n_{0}-2,1\right) v_{+}\left(n_{0}-1\right)+R(1)+\sum_{j=0}^{n_{0}} R\left(n_{0}-j\right)^{t} n_{+}\left(j, n_{0}-1-j\right)\right)^{t} \delta_{-}\left(n_{0}\right) \\
=\left(v_{-}\left(n_{0}\right)-v_{-}\left(n_{0}-1\right)^{t} n_{-}\left(n_{0}-1,1\right)+\delta_{-}\left(n_{0}\right) \delta_{+}\left(n_{0}\right) v_{-}\left(n_{0}-1\right)^{t} n_{-}\left(n_{0}-2,1\right)-\right. \\
-n_{0}^{-2}\left(n_{0}\right)\left(\sum_{j=0} n_{+}\left(j, n_{0}-1-j\right) R(1+j)+R\left(n_{0}\right)\right)^{t} \delta_{+}\left(n_{0}-1\right)^{t} \delta_{-}\left(n_{0}\right) .
\end{aligned}
$$

Therefore, it follows from Theorem 3.1 , Lemma 4.2 (i) and (4.10) that

$$
\begin{aligned}
\mathbb{I I}_{n_{0}+1}^{(1)}= & -v_{-}\left(n_{0}\right)^{t}\left(n_{-}\left(n_{0}-1,1\right)-n_{-}\left(n_{0}-2,1\right)\right)- \\
& -v_{-}\left(n_{0}-1\right)^{t} \delta_{+}\left(n_{0}-1\right)^{t} \delta_{-}\left(n_{0}\right)+ \\
& +\delta_{-}\left(n_{0}\right) \delta_{+}\left(n_{0}\right) v_{-}\left(n_{0}-1\right)^{t} \delta_{+}\left(n_{0}-1\right)^{t} \delta_{-}\left(n_{0}\right) \\
= & \left(v_{-}\left(n_{0}\right)-v_{-}\left(n_{0}-1\right)\right)^{t} \delta_{+}\left(n_{0}-1\right)^{t} \delta_{-}\left(n_{0}\right)+ \\
& +\delta_{-}\left(n_{0}\right) \delta_{+}\left(n_{0}\right) v_{-}\left(n_{0}-1\right)^{t} \delta_{+}\left(n_{0}-1\right)^{t} \delta_{-}\left(n_{0}\right) \\
= & 0 .
\end{aligned}
$$

By Steps 9 and 10 , we have proved that (*) ${ }_{n_{0}, 1}$ holds. Moreover, for any fixed $k, 1 \leq k<k+1 \leq N_{0}$, by assuming that (*) $\dot{n}_{0}, k$ holds, we will show that $(*) \dot{n}_{0}, k+1$ holds.

( Step 11) $\quad I_{n_{0}+1}^{(k+1)}=0$. 
Proof. By applying $\mathbb{I}_{n_{0}}^{(k)}=0\left(\right.$ resp. $\left.\mathbb{I I}_{n_{0}}^{(k)}=0\right)$ to the coefficient of $\delta_{+}\left(n_{0}-k\right)\left(\right.$ resp. $\left.\delta_{-}\left(n_{0}^{-k}\right)\right)$, we see from Theorem $3.1 \cdot,(4.8)$ and (4.9) that

$(4.12)$

$$
I_{n_{0}+1}^{(k+1)}=-\delta_{-}\left(n_{0}-k\right) I \cdot-I{ }^{t} \delta_{+}\left(n_{0}-k\right)+I \cdots
$$

where

$$
\begin{aligned}
& I^{\prime}=\sum_{j=0}^{k-1}\left(\sum_{i=0}^{n_{0}-2 k} n_{+}\left(i, n_{0}-k-i\right) R(k-j+j)\right)^{t} n_{+}\left(j, n_{0}-k-j\right)- \\
& \mathrm{k}-1 \\
& -\sum_{j=1}^{k}\left(n_{+}\left(n_{0}-k-j, j\right)-n_{+}\left(n_{0}-k-1-j, j\right)\right) \text {. } \\
& \cdot\left(R(k-j)+\sum_{i=1}^{n_{0}-2 k} R(k-j+i)^{t} n_{+}\left(n_{0}-k-i, i\right)\right)- \\
& -\left(n_{+}\left(n_{0}-2 k, k\right)-n_{+}\left(n_{0}-2 k-1, k\right)\right) v_{+}\left(n_{0}-k\right)+ \\
& +\delta_{+}\left(n_{0}-k\right) v_{-}\left(n_{0}-k-1\right)^{t} n_{+}\left(k-1, n_{0}-2 k\right) \text {, } \\
& I^{\prime \prime}=-\sum_{j=0}^{k-1} n_{-}\left(j, n_{0}-k-j\right)\left(\sum_{i=0}^{n_{0}-2 k} R(k-j+i)^{t} n_{-}\left(1, n_{0}-k-i\right)\right)+ \\
& +\sum_{j=1}^{k-1}\left(R(k-j)+\sum_{i=1}^{n_{0}-2 k} n_{-}\left(n_{0}-k-1,1\right) R(k-j+i)\right) . \\
& \cdot{ }^{t}\left(n_{-}\left(n_{0}-k-j, j\right)-n_{-}\left(n_{0}-k-1-j, j\right)\right)+ \\
& +v_{-}\left(n_{0}-k\right)^{t}\left(n_{-}\left(n_{0}-2 k, k\right)-n_{-}\left(n_{0}-2 k-1, k\right)\right)- \\
& -n_{-}\left(k-1, n_{0}-2 k\right) v_{+}\left(n_{0}-k-1\right){ }^{t} \delta_{-}\left(n_{0}-k\right) \\
& I^{\prime \prime \prime}=-\sum_{j=0}^{k-2} n_{-}\left(j, n_{0}-k-1-j\right)\left(R(k-1-j)+\sum_{i=1}^{n_{0}-2 k} R(k-1-j+i)^{t} n_{+}\left(n_{0}-k-i, i\right)\right)+ \\
& +\sum_{j=0}^{k-2}\left(R(k-1-j)+\sum_{i=1}^{n_{0}-2 k} n_{-}\left(n_{0}-k-1, i\right) R(k-1-j+i)\right)^{t} n_{+}\left(j, n_{0}-k-1-j\right)- \\
& -n_{-}\left(k-1, n_{0}-2 k\right) v_{+}\left(n_{0}-k-1\right)+v_{-}\left(n_{0}-k-1\right)^{t} n_{+}\left(k-1, n_{0}-2 k\right) \text {. }
\end{aligned}
$$

By Theorem 3.1 , and $I_{n-1}^{(k)}=0$,

(4.13) I"'

$$
\left.=\left(-\sum_{j=0}^{k-2} n_{-}\left(j, n_{0}-k-1-j\right) \sum_{i=0}^{n_{0}-2 k-1} R(k-j+i){ }^{t} n_{-}\left(i, n_{0}-k-1-i\right)\right)\right)^{t} \delta_{+}\left(n_{0}-k\right)+
$$




$$
+\delta_{-}\left(n_{0}-k\right)\left\{\sum_{j=0}^{k-2}\left(\sum_{i=0}^{n_{0}-2 k-1} n_{+}\left(i, n_{0}-k-1-i\right) R(k-j+i)\right)^{t} n_{+}\left(j, n_{0}-k-1-j\right)\right\}
$$

Next, applying Theorem $3.1^{\prime}$ to $I^{\prime}$, we have

$$
I^{\prime}=\delta_{+}\left(n_{0}-k\right) \tilde{I} \cdot{ }^{t} \delta_{-}\left(n_{0}-k\right)+\delta_{+}\left(n_{0}-k\right) \tilde{I I}^{\prime}+\tilde{I I I}^{\cdot t} \delta_{+}\left(n_{0}-k\right)+I V^{\prime} \text {, }
$$

where

$$
\begin{aligned}
& \tilde{I}^{\prime}=R(k)+\sum_{i=1}^{n_{0}-2 k} n_{-}\left(n_{0}-k-1-i, i\right) R(k+i)+ \\
& +n_{-}\left(k-1, n_{0}-2 k\right) v_{+}\left(n_{0}-k-1\right)^{t} \delta_{-}\left(n_{0}-k\right)+ \\
& +\sum_{j=1}^{k-1}\left(R(k-j)+\sum_{i=1}^{n_{0}-2 k} n_{-}\left(n_{0}-k-1-i, i\right) R(k-j+i)\right)^{t} n_{-}\left(n_{0}-k-1-j, j\right)- \\
& -\sum_{j=0}^{k-2} n_{-}\left(j, n_{0}-k-1-j\right)\left(R(k-j)+\sum_{i=0}^{n_{0}-2 k-1} R(k-j+i)^{t} n_{-}\left(i, n_{0}-k-i\right)\right) \\
& \tilde{I}^{\prime}=-n_{-}\left(k-1, n_{0}-2 k\right) v_{+}\left(n_{0}-k-1\right)+v_{-}\left(n_{0}-k-1\right)^{t} n_{+}\left(k-1, n_{0}-2 k\right)- \\
& -\sum_{j=0}^{k-2} n_{-}\left(j, n_{0}-k-1-j\right)\left(R(k-1-j)+\sum_{i=1}^{n_{0}-2 k} R(k-1-j+i)^{t} n_{+}\left(n_{0}-k-1-i, i\right)\right)+ \\
& +\sum_{j=0}^{k-2}\left(R(k-1-j)+\sum_{i=1}^{n_{0}-2 k} n_{-}\left(n_{0}-k-1-i, i\right) R(k-1-j+i)\right)^{t} n_{+}\left(j, n_{0}-k-1-j\right) \\
& \mathrm{n}_{0}-2 \mathrm{k}-1 \\
& \text { III. } \sum_{j=0} n_{+}\left(j, n_{0}-k-1-j\right) R(k+1+j)+ \\
& +\sum_{j=1}^{k-1}\left(\sum_{i=0}^{n_{0}-2 k-1} n_{+}\left(i, n_{0}-k-1-i\right) R(k-j+i)\right)^{t} n_{+}\left(n_{0}-k-1-j, j\right) \\
& I V^{\prime}=\sum_{j=0}^{k-2}\left(\sum_{i=0}^{n_{0}-2 k-1} n_{+}\left(i, n_{0}-k-1-i\right) R(k-j+1)\right)^{t} n_{+}\left(j, n_{0}-k-1-j\right) \text {. }
\end{aligned}
$$

By Lemma $4.2(1 \mathrm{i})$ and $\pi_{n_{0}-1}^{(k)}=0$,

$$
\begin{aligned}
\tilde{I}^{\prime}= & n_{-}\left(k-1, n_{0}-2 k\right)\left(R\left(n_{0}-k\right)+{ }^{n_{0}} \sum_{j=0}^{-2 k-2} R(1+j)^{t} n_{-}\left(j, n_{0}-k-1-j\right)+\right. \\
& \left.+v_{+}\left(n_{0}-k-1\right)^{t} \delta_{-}\left(n_{0}-k\right)\right)+I_{n_{0}-1}^{(k)}-v_{+}\left(n_{0}-k-1\right)^{t} n_{-}\left(n_{0}-2 k-1,1\right) \\
= & -v_{+}\left(n_{0}-k-1\right)^{t} n_{-}\left(n_{0}-2 k-1,1\right) .
\end{aligned}
$$

Furthermore, since 
(4.14)

$$
\tilde{I I}^{\prime}=I_{\mathrm{n}_{0}-1}^{(\mathrm{k})}=0 \text {. }
$$

$$
\begin{aligned}
I^{\prime}= & -\delta_{+}\left(n_{0}-k\right) v_{-}\left(n_{0}-k-1\right)^{t} n_{-}\left(n_{0}-2 k-1, k\right)^{t} \delta_{+}\left(n_{0}-k\right)+ \\
& +\tilde{I I I} \cdot{ }^{t} \delta_{+}\left(n_{0}-k\right)+\tilde{I} v .
\end{aligned}
$$

Similarly to (4.13), we can apply $I_{n_{0}-1}^{(k)}=\underset{n_{0}-1}{(k)}=0$ to see that

$$
\begin{aligned}
\text { II' }^{\prime}= & \delta_{-}\left(n_{0}-k\right) n_{+}\left(n_{0}-2 k-1, k\right) v_{+}\left(n_{0}-k-1\right)^{t} \delta_{-}\left(n_{0}-k\right)+ \\
& +\delta_{-}\left(n_{0}-k\right) \tilde{I}{ }^{\prime \prime}+\tilde{I V} "
\end{aligned}
$$

where

$$
\begin{aligned}
\tilde{I I I "}= & -\sum_{i=0}^{n_{0}^{-2 k-1}} R(k+i)^{t} n_{-}\left(i, n_{0}-k-1-i\right)- \\
& -\sum_{j=1}^{k-1} n_{+}\left(n_{0}-k-1-j, j\right)\left(\sum_{i=0}^{n^{-2 k-1}} R(k+1-j+i)^{t} n_{-}\left(i, n_{0}-k-1-i\right)\right) \\
\tilde{\text { IV" }}= & -\sum_{j=0}^{k-2} n_{-}\left(j, n_{0}-k-1-j\right)\left(\sum_{i=0}^{-2 k-1} R(k-j+i)^{t} n_{-}\left(i, n_{0}-k-1-i\right)\right) .
\end{aligned}
$$

Thus, by $(4.12),(4.13),(4.14)$ and $(4.15)$,

(4.16)

$$
\begin{aligned}
& I_{n_{0}+1}^{(k+1)}=-\delta_{-}\left(n_{0}-k\right)\left(-\delta_{+}\left(n_{0}-k\right) v_{-}\left(n_{0}-k-1\right)^{t} n_{-}\left(n_{0}-2 k-1, k\right)+\right. \\
& \left.\quad+n_{+}\left(n_{0}-2 k-1, k\right) v_{+}\left(n_{0}-k-1\right){ }^{t} \delta_{-}\left(n_{0}-k\right)+\tilde{I I I} \cdot+\tilde{I I I}\right)^{t} \delta_{+}\left(n_{0}-k\right) .
\end{aligned}
$$

On the other hand, by using $I V_{n_{0}-1}^{(k)}=0$, coming from $\quad(*)_{n_{0}}$ and step

5, we see that

$$
\begin{aligned}
\tilde{I I I}^{\prime}+\tilde{\text { III }}= & n_{+}\left(n_{0}-2 k-1, k\right)\left(R\left(n_{0}-k\right)+\sum_{j=0}^{n_{0}-k-2} R(1+j)^{t} n_{-}\left(j, n_{0}-k-1-j\right)\right)- \\
& -\left(R\left(n_{0}-k\right)+\sum_{j=0}^{n_{0}-k-2} n_{+}\left(j, n_{0}-k-1-j\right) R(1+j)\right)^{t} n_{-}\left(n_{0}-2 k-1, k\right) .
\end{aligned}
$$

Consequently, by substituting this into (4.16), it follows from Lemma 4.2 that $I_{n_{0}+1}^{(k+1)}=0$.

$$
(\underline{\text { Step }} 12) \quad \text { (i) } \quad \pi_{n_{0}+1}^{(k+1)}=0 \quad \text { (i i ) } \quad \pi_{n_{0}+1}^{(k+1)}=0 \text {. }
$$

Proof. We prove only (i), since (ii) is shown similarly. By applying $\quad \mathrm{IV}_{\mathrm{n}_{0}}^{(\mathrm{k})}=0$ (resp. $\mathbb{I}_{n_{0}}^{(k)}=0$ ) to the coefficient of $\delta_{-}\left(n_{0}-k\right)$ 
(resp. $\left.{ }^{t} n_{-}\left(n_{0}-k-1,1\right)\right)$ in $\pi_{n_{0}+1}^{(k+1)}$, we see from Theorem 3.1' that (4.17) $\quad$ III $_{n_{0}+1}^{(k+1)}=v_{-}\left(n_{0}-k\right)^{t}\left(n_{-}\left(n_{0}-2 k-1, k+1\right)-n_{-}\left(n_{0}-k-1,1\right) n_{-}\left(n_{0}-2 k, k\right)\right)$ $+\left(I-\delta_{-}\left(n_{0}-k\right) \delta_{+}\left(n_{0}-k\right)\right) J_{1}-\delta_{-}\left(n_{0}-k\right) J_{2}-J_{3}^{t} n_{-}\left(n_{0}-k-1,1\right)+J_{4}$

where

$$
\begin{aligned}
& J_{1}=R(k+1)+\sum_{i=1}^{n_{0}-2 k-1} n_{-}\left(n_{0}-k-1-i, i\right) R(k+1+i)- \\
& -\sum_{j=0}^{k-1} n_{-}\left(j, n_{0}-k-1-j\right)\left(\sum_{i=0}^{n_{0}-2 k-1} R(k-j+i)^{t} n_{-}\left(i, n_{0}-k-i\right)\right) \\
& J_{2}=\sum_{j=1}^{k}\left(\sum_{i=0}^{n_{0}-2 k-1} n_{+}\left(i, n_{0}-k-i\right) R(k+1-j+i)\right)^{t} n_{-}\left(n_{0}-k-j, j\right) \\
& J_{3}=-\sum_{j=0}^{k-1} n_{-}\left(j, n_{0}-k-j\right)\left(\sum_{i=0}^{n_{0}-2 k-1} R(k-j+i)^{t} n_{-}\left(i, n_{0}-k-i\right)\right)- \\
& +\sum_{j=1}^{k-1}\left(R(k-j)+\sum_{i=1}^{n_{0}-2 k} n_{-}\left(n_{0}-k-i, i\right) R(k+1+i)\right)^{t} n_{-}\left(n_{0}-k-j, j\right)+ \\
& +n_{-}\left(k, n_{0}-2 k\right) R\left(n_{0}-k\right) \\
& J_{4}=\sum_{j=2}^{k}\left(R(k+1-j)+\sum_{i=1}^{n_{0}-2 k-1} n_{-}\left(n_{0}-k-i, i\right) R(k+1-j+i)\right)^{t} n_{-}\left(n_{0}-k-j, j\right) .
\end{aligned}
$$

Since

$$
\begin{aligned}
J_{3} & =\pi_{n_{0}}^{(k)}-\left(R(k)+\sum_{i=1}^{n_{0}-2 k} n_{-}\left(n_{0}-k-i, i\right) R(k+i)\right)- \\
& -v_{-}\left(n_{0}-k\right)^{t} n_{-}\left(n_{0}-2 k, k\right)+n_{-}\left(k, n_{0}-k\right) R\left(n_{0}-k\right),
\end{aligned}
$$

it follows from Theorem 3.1' that

$$
\begin{aligned}
J_{3}= & -\delta_{-}\left(n_{0}-k\right)\left(\sum_{i=0}^{n_{0}-2 k-2} n_{+}\left(i, n_{0}-k-1-i\right) R(k+1+i)\right)- \\
& -\left(R(k)+n_{0}^{-2 k-1} \sum_{i=1} n_{-}\left(n_{0}-k-1-1,1\right) R(k+i)\right)-v_{-}\left(n_{0}-k\right)^{t} n_{-}\left(n_{0}-2 k, k\right)
\end{aligned}
$$

and so

$(4.18)$

$$
\begin{aligned}
& \delta_{-}\left(n_{0}-k\right) J_{2}+J_{3}{ }^{t} n_{-}\left(n_{0}-k-1,1\right) \\
= & -\left(1-\delta_{-}\left(n_{0}-k\right) \delta_{+}\left(n_{0}-k\right)\right)(R(k)+
\end{aligned}
$$




$$
\begin{aligned}
& \left.\quad+\sum_{0}^{n_{0}-2 k-1} n_{-}\left(n_{0}-k-1-i, i\right) R(k+i)\right)^{t} n_{-}\left(n_{0}-k-1,1\right)+ \\
& +\delta_{-}\left(n_{0}-k\right) \delta_{+}\left(n_{0}-k\right) \sum_{j=2}^{k}(R(k+1-j)+ \\
& \left.+n_{0}^{-2 k-1} \sum_{i=1}^{i} n_{-}\left(n_{0}-k-1-i, i\right) R(k+1-j+i)\right)^{t} n_{-}\left(n_{0}-k-j, j\right)+ \\
& +\delta_{-}\left(n_{0}-k\right) \sum_{j=2}^{k}\left(\sum_{0}^{-2 k-2} \sum_{i=0}^{i} n_{+}\left(n_{0}-k-1-i, i\right) R(k+2-j+i)\right)^{t} n_{-}\left(n_{0}-k-j, j\right)- \\
& -v_{-}\left(n_{0}-k\right){ }^{t} n_{-}\left(n_{0}-2 k, k\right){ }^{t} n_{-}\left(n_{0}-k-1,1\right) .
\end{aligned}
$$

Forthermore, we note from Theorem $3.1^{\prime}$ that

$$
\text { (4.19) J } \mathrm{J}_{4}
$$

$$
\begin{aligned}
= & \sum_{j=2}^{k}\left(R(k+1-j)+{ }^{n_{0}-2 k-1} \sum_{i=1}^{i} n_{-}\left(n_{0}-k-1-1, i\right) R(k+1-j+j)\right)^{t} n_{-}\left(n_{0}-k-j, j\right)+ \\
& +\delta_{-}\left(n_{0}-k\right) \sum_{j=2}^{k}\left({ }^{n} \sum_{i=0}^{-2 k-2} n_{+}\left(n_{0}-k-1-i, i\right) R(k+2-j+1)\right)^{t} n_{-}\left(n_{0}-k-j, j\right) .
\end{aligned}
$$

Therefore, by using Theorem $3.1^{\prime}$ and (4.9), we see from (4.17), (4.18) and (4.19) that

$$
\text { (4.20) } \quad \pi_{n_{0}+1}^{(k+1)}=\left(1-\delta_{-}\left(n_{0}-k\right) \delta_{+}\left(n_{0}-k\right)\right) J_{5} \text {, }
$$

where

$$
\begin{aligned}
J_{5} & =R(k+1)+{ }^{n_{0}-2 k-2} \sum_{i=1} n_{-}\left(n_{0}-k-1-i, i\right) R(k+1+i)+ \\
& +\sum_{j=1}^{k}\left(R(k+1-j)+\sum_{0}^{-2 k-2} \sum_{i=1} n_{-}\left(n_{0}-k-1-i, i\right) R(k+1-j+i)\right)^{t} n_{-}\left(n_{0}-k-j, j\right)- \\
& -\sum_{j=0}^{k-1} n_{-}\left(j, n_{0}-k-1-j\right)\left(R(k-j){ }^{t} \delta_{-}\left(n_{0}-k\right)+\sum_{0}^{-2 k-1} R(k-j+i) n_{-}\left(i, n_{0}-k-i\right)\right)+ \\
& +n_{-}\left(k, n_{0}-2 k-1\right)\left(R\left(n_{0}-k\right)+\sum_{i=1}^{k} R\left(n_{0}-k-i\right){ }^{t} n_{-}\left(n_{0}-k-i, i\right)\right)+ \\
& +v_{-}\left(n_{0}-k-1\right){ }^{t} n_{-}\left(n_{0}-2 k-1, k+1\right) .
\end{aligned}
$$

We note from Lemma 4.2 (ii) that 


$$
\begin{aligned}
& R\left(n_{0}-k\right)+\sum_{i=1}^{k} R\left(n_{0}-k-i\right)^{t} n_{-}\left(n_{0}-k-1-i, i\right) \\
= & -v_{+}\left(n_{0}-k-1\right)^{t} \delta_{+}\left(n_{0}-k\right)-\sum_{0}^{n_{0}-2 k-2} R(1+i)^{t} n_{-}\left(i, n_{0}-k-1-i\right) .
\end{aligned}
$$

Therefore, by using Theorem 3.1', we get

$$
\begin{aligned}
& (4.21)^{\mathrm{J}} 5 \\
& =R(k+1)+\sum_{i=1}^{n_{0}-2 k-2} n_{-}\left(n_{0}-k-1-i, i\right) R(k+1+i)-v_{-}\left(n_{0}-k-1\right)^{t} n_{-}\left(n_{0}-2 k-2, k+1\right)+ \\
& +\sum_{j=1}^{k}\left(R(k+1-j)+\sum_{i=1}^{n_{0}-2 k-2} n_{-}\left(n_{0}-k-1-i, i\right) R(k+1-j+i)\right)^{t} n_{-}\left(n_{0}-k-1-j, j\right)- \\
& -\sum_{j=0}^{k} n_{-}\left(j, n_{0}-k-1-j\right)\left(\sum_{i=0}^{n_{0}-2 k-2} R(k-j+1+1)^{t} n_{-}\left(i, n_{0}-k-1-i\right)\right)+ \\
& +1 \sum_{j=1}^{k}\left(R(k+1-j)+\sum_{i=1}^{n_{0}-2 k-2} n_{-}\left(n_{0}-k-1-i, i\right) R(k+1-j+i)\right)^{t} n_{+}\left(j-1, n_{0}-k-j\right)-
\end{aligned}
$$

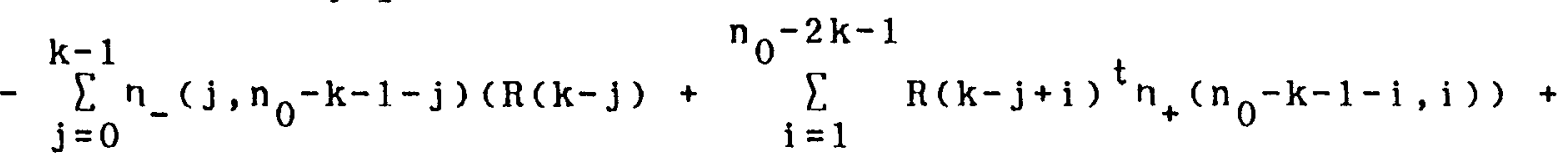

$$
\begin{aligned}
& +n_{-}\left(k, n_{0}-2 k-1\right)\left(-v_{+}\left(n_{0}-k-1\right)+\sum_{i=1}^{k} R\left(n_{0}-k-i\right) t_{+}\left(i-1, n_{0}-k-i\right)\right)+ \\
& \left.+v_{-}\left(n_{0}-k-1\right)^{t} n_{+}\left(k, n_{0}-2 k-1\right)\right)^{t} \delta_{-}\left(n_{0}-k\right) \\
& =I_{n_{0}}^{(k+1)}+I_{n_{0}}^{(k+1) t} \delta_{-}\left(n_{0}-k\right) \\
& =0 \text {, }
\end{aligned}
$$

noting that $k+1 \leq N_{0}$. Consequently, by (4.20) and (4.21), we see that $\Pi_{n_{0}+1}^{(k+1)}=0$

Finally, we will show $(*){ }_{n_{0}}, N_{0}$, that is,

$$
\left(\begin{array}{l}
\text { Step 13) } \\
\text { I }
\end{array} \operatorname{lv}_{n_{0}+1}^{\left(N_{0}\right)}=0\right. \text {. }
$$

Proof. Since it follows from (4.8) and (4.9) that

$$
v_{+}\left(N_{0}\right)+\sum_{j=0}^{N_{0}-1} v_{+}\left(N_{0}-1-j\right)^{t} \delta_{-}\left(N_{0}-j\right)^{t} \delta_{+}\left(N_{0}-j\right)
$$




$$
\begin{aligned}
& =v_{-}\left(N_{0}\right)+\sum_{j=0}^{N_{0}-1} \delta_{-}\left(N_{0}-j\right) \delta_{+}\left(N_{0}-j\right) v_{-}\left(N_{0}-1-j\right) \\
& =R(0),
\end{aligned}
$$

we see from (ii) in step 6 that $\mathrm{Step} 13$ holds.

(Q.E.D.)

Thus we have completed the proof of Lemma 4.3. By noting Steps 1,2 and 3 in the proof of Lemma 4.3, we have

Theorem 4.1. For any $n \in \mathbb{N}^{*}$,

$$
v_{+}(n+1)=v_{+}(n)-\delta_{+}(n+1) v_{-}(n)^{t} \delta_{+}(n+1)
$$

$$
v_{+}(n+1)=\left(1-\delta_{+}(n+1) \delta_{-}(n+1)\right) v_{+}(n)
$$

(iv)

$$
v_{-}(n+1)=v_{-}(n)-\delta_{-}(n+1) v_{+}(n)^{t} \delta_{-}(n+1)
$$

( v )

( vi )

$$
\begin{aligned}
& v_{-}(n+1)=\left(I-\delta_{-}(n+1) \delta_{+}(n+1)\right) V_{-}(n) \\
& v_{+}(n)^{t} \delta_{-}(n+1)=\delta_{+}(n+1) V_{-}(n)
\end{aligned}
$$

$$
v_{+}(n+1)^{t} \delta_{-}(n+1)=\delta_{+}(n+1) v_{-}(n+1) \text {. }
$$

By Theorems 3.1 and 4.1 , we find that the parameters $\gamma_{+}(\cdot, *)$, $\gamma_{-}(\cdot, *), V_{+}(\cdot)$ and $V_{-}(\cdot)$ in $\mathrm{KM}_{2} \mathrm{O}$-Langevin equations (2.16) and (2.17) can be uniquely determined by $R(0), \delta_{+}(\cdot)$ and $\delta_{-}(\cdot)$. We call the system $\left(\gamma_{+}(n, k), \delta_{+}(m), v_{+}(m) ; m, n, k \in \mathbb{N}, n>k\right\}$ (resp. $\left(\gamma_{-}(n, k)\right.$, $\left.\left.\delta_{-}(m), V_{-}(m) ; m, n, k \in \mathbb{N}, n>k\right\}\right)$ the forward (resp. backward) $K_{2}$ o-Langevin data associated with X.

For future use in a modelling of time series, we will rewrite Lemma 4.2 into

Theorem 4.2. For any $n \in \mathbb{N}^{*}$,

$$
\begin{aligned}
& \delta_{+}(n+1)=-\left(R(n+1)+\sum_{k=0}^{n-1} \gamma_{+}(n, k) R(k+1)\right) v_{-}(n)^{-1} \\
& \delta_{-}(n+1)=-\left({ }^{t} R(n+1)+\sum_{k=0}^{n-1} \gamma_{-}(n, k){ }^{t} R(k+1)\right) v_{+}(n)^{-1} .
\end{aligned}
$$




\section{\$5. The prediction formula and prediction error}

For each $m, n \in \mathbb{N}, m \geq n$, we define a forward (resp. backward) prediction matrix $P_{+}(m, n)\left(\right.$ resp. $\left.P_{-}(m, n)\right) \in M(d ; \mathbb{R})$ and a forward (resp.backward) prediction error matrix $e_{+}(m, n)\left(r e s p . e_{-}(m, n)\right) E$ $M(d ; \mathbb{R})$ by

$$
P_{+}(m, n)=E\left(X(m)^{t} I_{+}(n)\right) v_{+}(n)^{-1 / 2}
$$

$$
P_{-}(m, n)=E\left(X(-m)^{t} I_{-}(n)\right) v_{-}(n)^{-1 / 2}
$$

$$
\begin{aligned}
& e_{+}(m, n)=E\left(\left(X(m)-P M_{0}^{+}(n) X(m)\right)^{t}\left(X(m)-P M_{0}^{+}(n) X(m)\right)\right) \\
& e_{-}(m, n)=E\left(\left(X(-m)-P_{M_{0}^{-}(n)} X(-m)\right)^{t}\left(X(-m)-P M_{0}^{-}(n) X(-m)\right)\right) .
\end{aligned}
$$

Theorem 5.1. For any $m, n \in \mathbb{N}, m \geq n$,

$$
\begin{aligned}
& X(m)=R(m) R(0)^{-1} X(0)+\sum_{k=1}^{m} P_{+}(m, k) v_{+}(k)^{-1 / 2} I_{+}(k) \\
& P_{M_{0}^{+}(n)} X(m)=R(m) R(0)^{-1} X(0)+\sum_{k=1}^{n} P_{+}(m, k) V_{+}(k)^{-1 / 2} I_{+}(k)
\end{aligned}
$$

$$
e_{+}(m, n)=\sum_{k=n+1}^{m} P_{+}(m, k){ }^{t} P_{+}(m, k) \text {. }
$$

Proof. We put

$W=$ the left hand side in (i) - the right hand side in (i). By (2.20), we see that each component of $W$ belongs to the linear hull of $\{I+j(l) ; 1 \leq j \leq d, 0 \leq \ell \leq m\}$. It follows from (2.18) and (5.1) that $E\left(W^{t} I_{+}(l)\right)=0(0 \leq l \leq m)$. Hence, we find that $W=0$, implying (i). (ii) follows from (2.18), (2.20) and (i). By noting (2. 18) again, we see that ( $1 \mathrm{i} i$ ) follows from (i) and (ii). (Q.E.D.)

\footnotetext{
Similarly, we obtain
}

Theorem 5.2. For any $m, n \in \mathbb{N}, m \geq n$, 
(i)

$$
X(-m)={ }^{t} R(m) R(0)^{-1} X(0)+\sum_{k=1}^{m} P_{-}(m, k) v_{-}(k)^{-1 / 2} I_{-}(k)
$$

( $\mathrm{i}$ i )

$$
P_{M_{0}^{-}(n)} X(-m)={ }^{t} R(m) R(0)^{-1} X(0)+\sum_{k=1}^{n} P_{-}(m, k) V_{-}(k)^{-1 / 2} I_{-}(k)
$$

$$
e_{-}(m, n)=\sum_{k=n+1}^{m} P_{-}(m, k)^{t} P_{-}(m, k) \text {. }
$$

We will show that the prediction matrices $\mathrm{P}_{+}(\cdot, *)$ and $P_{-}(\cdot, *)$ can be also determined by $R(0), \delta_{+}(\cdot)$ and $\delta_{-}(\cdot)$ through the following

Theorem 5.3. For any $m, n \in N, m>n$,

$$
\begin{aligned}
& P_{+}(n, n)=v_{+}(n) 1 / 2 \\
& P_{+}(m, n)=-\sum_{k=n}^{m-1} \gamma_{+}(m, k) P_{+}(k, n) \\
& P_{-}(n, n)=v_{-}(n) 1 / 2 \\
& P_{-}(m, n)=-\sum_{k=n}^{m-1} \gamma_{-}(m, k) P_{-}(k, n) .
\end{aligned}
$$

Proof. By $(2.16),(2.18),(2.20)$ and $(5.1), P_{+}(n, n)=v_{+}(n)^{1 / 2}$. By substituting (2.16) replaced $n$ by $m$ into the right hand side of (5.1), we find from (2.18) and (2.20) that the second statement in (i) holds. (ii) is also proved similarly.

By using Theorem 4.1 (i i)( $1 v$ ), we see from Theorems 5.1 (ii i), 5.2 (i i a ) 5.3 that the one-step prediction error matrices $e_{+}(n+1, n)$ and $e_{-}(n+1, n)$ can be explicitly represented by $R(0), \delta_{+}(\ell)$ and $\delta_{-}(\ell)(1 \leq \ell \leq n+1)$ through the following

Theorem 5.4. For any $n \in \mathbb{N}$,

$$
\begin{aligned}
& e_{+}(n+1, n)=\left(I-\delta_{+}(n+1) \delta_{-}(n+1)\right) \cdots\left(I-\delta_{+}(1) \delta_{-}(1)\right) R(0) \\
& e_{-}(n+1, n)=\left(I-\delta_{-}(n+1) \delta_{+}(n+1)\right) \cdots\left(I-\delta_{-}(1) \delta_{+}(1)\right) R(0) .
\end{aligned}
$$




\section{A construction theorem}

As a converse setting of $\$ 2 \sim \S 5$, we are given a system $\left(V, \delta_{+}(n)\right.$; $n \in \mathbb{N}\}$ of members in $M(d ; \mathbb{R})$ such that

(6.1) $\quad V$ is a symmetric positive definite

$$
\delta_{+}(n) \in M(d ; \mathbb{R}) \quad(n \in \mathbb{N}) .
$$

Then we construct a triple $\left(v_{+}(1), \delta_{-}(1), v_{-}(1)\right)$ by

$$
\left\{\begin{array}{l}
v_{+}(1)=v-\delta_{+}(1) v^{t} \delta_{+}(1) \\
\delta_{-}(1)=v^{t} \delta_{+}(1) v^{-1} \\
v_{-}(1)=v-\delta_{-}(1) v^{t} \delta_{-}(1) .
\end{array}\right.
$$

In order to continue the following construction of

$$
\begin{gathered}
\left(v_{+}(n), \delta_{-}(n), v_{-}(n)\right) \text { from }\left(v_{+}(n-1), \delta_{-}(n-1), v_{-}(n-1)\right)(n \in \mathbb{N}): \\
(6.4)\left\{\begin{array}{l}
v_{+}(n)=v_{+}(n-1)-\delta_{+}(n) v_{-}(n-1){ }^{t} \delta_{+}(n) \\
\delta_{-}(n) v_{+}(n-1)=v_{-}(n-1) t \delta_{+}(n) \\
v_{-}(n)=v_{-}(n-1)-\delta_{-}(n-1) v_{+}(n-1){ }^{t} \delta_{-}(n-1),
\end{array}\right.
\end{gathered}
$$

we suppose that

(6.5)

$$
V_{+}(n-1) \in G L(d ; \mathbb{R}) \quad(n \in \mathbb{N}) \text {, }
$$

where $v_{+}(0)=v_{-}(0)=v$.

Next, we construct a system $\left(\gamma_{+}(m, n), \gamma_{-}(m, n) ; m, n \in \mathbb{N}^{*}, m>n\right)$ of members in $M(d ; R)$ according to the algorithm in Theorem 3.1 .

Furthermore, we prepare any $\mathbb{R}^{d}$-valued stochastic process $I_{+}=$ $\left(I_{+}(n) ; n \in \mathbb{N}^{*}\right)$ on a probability space $(\Omega, B, P)$ such that

$$
\mathrm{E}\left(I_{+}(\mathrm{n})\right)=0
$$$$
\left(n \in \mathbb{N}^{*}\right)
$$

$$
E\left(I_{+}(n)^{t} I_{+}(m)\right)=\delta_{n m} V_{+}(n)
$$$$
\left(n, m \in \mathbb{N}^{*}\right)
$$

and then construct an $\mathbb{R}^{d}$-valued stochastic process $X_{+}=\left(X(n) ; n \in \mathbb{N}^{*}\right)$ by

$$
(6.8) \quad X(0)=I_{+}(0)
$$


$(6.9)$

$$
X(n)=-\sum_{k=0}^{n-1} \gamma_{+}(n, k) X(k)+I_{+}(n) \quad(n \in \mathbb{N}) .
$$

Now we will show

Theorem 6.1. $X_{+}$is weakly stationary.

For any $m, n \in \mathbb{N}^{*}$, we put

(6.10) $\quad R(m, n)=E\left(X(m)^{t} X(n)\right)$.

Since

(6.11) $\quad t_{R(m, n)}=R(n, m)$.

for the proof of Theorem 6.1, it suffices to show

(6.12) $R(m+l, n+l)=R(m, n)(m \geq n \geq 0, \ell \geq 1)$.

The proof is divided into 22 steps.

(

This follows immediately from (6.7), (6.8) and (6.9).

(step 2) (i) $\quad \mathrm{R}(0,0)=\mathrm{R}(1,1)=\mathrm{R}(2,2)=\mathrm{V}$

(i i)

$$
R(1,0)=R(2,1)=-\delta_{+}(1) \mathrm{V}
$$

$$
R(2,0)=\delta_{+}(1) \delta_{+}(1) v-\delta_{+}(2) v_{-}(1) \text {. }
$$

These are shown by a simple calculation.

(Step 3)

$$
\text { (i) } \delta_{-}(n+1) v_{+}(n)=v_{-}(n)^{t} \delta_{+}(n+1) \quad\left(n \in \mathbb{N}^{*}\right)
$$

$$
\delta_{-}(n) v_{+}(n)=v_{-}(n)^{t} \delta_{-}(n)
$$

$(n \in \mathbb{N})$.

Proof. (i) is included in (6.4) and (ii) is proved similarly to Step 7 in the proof of Lemma 4.3 . (Q.E.D.)

$$
\begin{aligned}
& \text { (Step 4) (i) } R(n, l-1)=-\sum_{k=0}^{n-1} n_{+}(k, n-k) R(k, l-1) \quad(n \geq l \geq 1) \\
& \begin{aligned}
& \text { (i i ) } \\
& R(n, n)=-\sum_{k=0}^{n-1} n_{+}(k, n-k) R(k, n)+v_{+}(n) \quad(n \geq 1) \\
& \text { (i i i ) } v_{+}(n)= R(n, n)+\sum_{k=1}^{n-1} n_{+}(k-1, n-k) R(k, n)+ \\
&+\delta_{+}(n)\left(\sum_{k=1}^{n-1} n_{-}(n-k-1, k) R(k, n)+R(0, n)\right) \quad(n \geq 2)
\end{aligned}
\end{aligned}
$$


where $\left(n_{+}(m, n), n_{-}(m, n) ; m \in \mathbb{N}^{*}, n \in \mathbb{N}\right) 1$ is defined by (3.6) and (3.7).

Proof. By multiplying both hand sides of (6.9) by ${ }^{t} X(l-1)$ (resp. ${ }^{t} X(n)$ ) and then taking an expectation with respect to the probability $P$, we have (i) (resp. (ii)). (iii) follows from(ii) and the algorithm in Theorem 3.1'.

(Q.E.D.)

( step 5$)$ (1) $R(n, 0)=-\delta_{+}(n) v_{-}(n-1)-\sum_{k=1}^{n-1} n_{+}(k-1, n-k) R(k, 0) \quad(n \in \mathbb{N})$ (i i) $\quad v_{-}(n-1)=R(0,0)+\sum_{k=1}^{n-1} R(0, k)^{t_{n}}(n-1-k, k) \quad$ (n $\left.\in \mathbb{N}\right)$ (i i i ) $R(n, 0)=-\delta_{+}(n) V-\sum_{k=1}^{n-1} n_{+}(k, n-k) R(k, 0)$ $(n \in \mathbb{N})$

Proof. By a mathematical induction about $n$, we show (i) and (ii). By Step 2, (i) and (ii) for $n=1$ hold. Let us assume that

(i) and (ii) hold for $n=n_{0}$. Since $v_{-}\left(n_{0}\right)$ is symmetric, by (6.4) (6.11) and (i) for $n=n_{0}$,

$$
\begin{aligned}
v_{-}\left(n_{0}\right) & =v_{-}\left(n_{0}-1\right)-v_{-}\left(n_{0}-1\right)^{t} \delta_{+}\left(n_{0}\right)^{t} \delta_{-}\left(n_{0}\right) \\
& =v_{-}\left(n_{0}-1\right)+\left(R\left(0, n_{0}\right)+\sum_{k=1} R(0, k)^{t} n_{+}\left(k-1, n_{0}-k\right)\right)^{t} \delta_{-}\left(n_{0}\right) .
\end{aligned}
$$

On the other hand, by Theorem 3.1',

$$
\begin{aligned}
R(0,0) & +\sum_{k=1}^{n} R(0, k)^{t} n_{-}\left(n_{0}-k, k\right) \\
=R(0,0) & +\sum_{k=1}^{n_{0}-1} R(0, k)^{t} n_{-}\left(n_{0}-1-k, k\right)+ \\
& +\left(R\left(0, n_{0}\right)+\sum_{k=1} R(0, k)^{t} n_{+}\left(k-1, n_{0}-k\right)\right)^{t} \delta_{-}\left(n_{0}\right) .
\end{aligned}
$$

Hence, we have (ii) for $n=n_{0}+1$. Next, by (6.9) and Theorem 3.1',

$$
R\left(n_{0}+1,0\right)=-\sum_{k=0}^{n_{0}} n_{+}\left(k, n_{0}+1-k\right) R(k, 0)
$$




$$
\begin{aligned}
& =-\sum_{k=1}^{n_{0}} n_{+}\left(k-1, n_{0}+1-k\right) R(k, 0)- \\
& -\delta_{+}\left(n_{0}+1\right)\left(R(0,0)+\sum_{k=1}^{n_{0}} n_{-}\left(n_{0}-k, k\right) R(k, 0)\right),
\end{aligned}
$$

which, together with (ii) for $n=n_{0}$, implies (1) for $n=n_{0}+1$. By substituting (ii) into (i), we see from Theorem $3.1^{\prime}$ that (iii) holds.

(Q.E.D.)

Before proceeding to the next step, we put the following statements: for each $n \in \mathbb{N}^{*}$.

$$
\begin{array}{ll}
(6.13)_{n} & \sum_{k=0}^{n-1} n_{+}(k, n-k) R(k+1,0)=\sum_{k=0}^{n-1} R(k+1,0) t_{-}(k, n-k) \\
(6.14)_{n} & v_{+}(n)=R(0,0)+\sum_{k=1}^{n} R(k, 0) n_{+}(n-k, k) \\
(6.15)_{n} & R(0, n)=-\delta_{-}(n) v_{+}(n-1)-\sum_{k=1}^{n-1} n_{-}(k-1, n-k) R(0, k) \\
(6.16)_{n} & R(0, n)=-\delta_{-}(n) v-\sum_{k=1}^{n-1} n_{-}(k, n-k) R(0, k) . \\
\text { (step 6) For any fixed } n \in \mathbb{N}, \\
\text { (i) }
\end{array}
$$

Proof. By (6.9) and Theorem 3.1',

$$
\begin{aligned}
R(0, n+1)= & -\sum_{k=0}^{n} R(0, k)^{t} n_{+}(k, n+1-k) \\
= & -\sum_{k=1}^{n} R(0, k)^{t} n_{+}(k-1, n+1-k)- \\
& -\left(R(0,0)+\sum_{k=1}^{n} R(0, k)^{t} n_{-}(n-k, k)\right)^{t} \delta_{+}(n+1) .
\end{aligned}
$$

And so by (ii) in step 5 ,

$$
R(0, n+1)=-t\left(\sum_{k=0}^{n-1} n_{+}(k, n-k) R(k+1,0)\right)-v_{-}(n)^{t} \delta_{+}(n+1) \text {. }
$$


Therefore, we have (i). ( $i$ i) and ( $i$ i $)$ are proved similarly as in the proof of ( $i$ i) and ( $i$ i i) in step 5.

We will show (6.13) $)_{n}$ and $(6.14)_{n}(n \in \mathbb{N})$ by a mathematical induction about $n$ in Step 7 Step 17. Immediately from (i) and (ii) in step $2,(6.13)_{1}$ and $(6.14)_{1}$ hold. For any fixed $n_{0} \in\{2,3, \cdots\}$, let us assume that $(6.13)_{\ell}$ and $(6.14)_{\ell}$ hold for any $\ell \in\left(1,2, \cdots, n_{0}-1\right)$. (Step 7) If $(6.13) \mathrm{n}_{0}$ holds, then $(6.14) \mathrm{n}_{0}$ does.

This follows from (i) and (ii) in step 6 .

(Step 8) (i) the left hand side of (6.13) $n_{0}=-\sum_{k=1}^{n_{0}} c_{n_{0}}(k)^{t} \delta_{-}(k)$ (ii) the right hand side of (6.13) $n_{0}=\sum_{k=1}^{n_{0}} D_{n_{0}}(k)^{t} \delta_{-}(k)$,

where

$$
\text { (6.17) } \begin{aligned}
C_{n_{0}}(k)= & \sum_{l=k}^{n_{0}-1} n_{+}\left(l, n_{0}-l\right)\left(R(l+1-k, 0)+\sum_{m=0}^{k-2} R(l-m, 0)^{t} n_{+}(m, k-1-m)\right)+ \\
+ & n_{+}\left(k-1, n_{0}-k+1\right) v_{+}(k-1) \\
(6.18) \quad D_{n_{0}}(k)= & R(n+1-k)+\sum_{m=0}^{k-2} R\left(n_{0}-m, 0\right)^{t} n_{+}(m, k-1-m) .
\end{aligned}
$$

Proof. Since it follows from (i) in step 6 that $(6.15)_{\ell}\left(1 \leq l \leq n_{0}\right)$ hold, we get

(6.19) the left hand side of (6.13) $n_{0}$

$$
\begin{aligned}
= & -\sum_{k=1}^{n_{0}-1} n_{+}\left(k, n_{0}-k\right)\left(v_{+}(k)^{t} \delta_{-}(k+1)+\sum_{l=1}^{k} R(l, 0)^{t} n_{-}(l-1, k+1-l)\right)- \\
& -\delta_{+}\left(n_{0}\right) v^{t} \delta_{-}(1) .
\end{aligned}
$$

By Theorem $3.1^{\prime}$,

$$
\begin{array}{ll}
\text { (6.20) } & n_{-}(l-1, k+1-l)=\delta_{-}(k+1-l)+\sum_{j=1}^{\ell-1} \delta_{-}(k+1-l+j) n_{+}(k-l, j) \\
(6.21) & n_{-}\left(k, n_{0}-k\right)=\delta_{-}\left(n_{0}-k\right)+\sum_{j=1}^{k} \delta_{-}\left(n_{0}-k+j\right) n_{+}\left(n_{0}-k-1, j\right) .
\end{array}
$$


By substituting (6.20) (resp. (6.21)) into (6.19) (resp. the right hand side of (6.13) $n_{0}$ ), we have (i) (resp. (i i )) in step 8 .

(Q.E.D.)

( step 9) For any $k \in\left\{1,2, \cdots, n_{0}\right\}$,

$$
C_{n_{0}}(k)+D_{n}(k)=\sum_{l=0}^{k-1} \delta_{+}\left(n_{0}-l\right) E_{n_{0}}(l ; k) \text {. }
$$

where for $\ell \in(0, \cdots, k-3)$,

$$
\text { (6.22) } \begin{aligned}
E_{n_{0}}(l ; k) & \\
= & \sum_{0} \sum_{i=0}^{-k-1} n_{-}\left(i, n_{0}-l-1-i\right) R\left(n_{0}-k-i, 0\right)+n_{-}\left(n_{0}-k, k-l-1\right) v_{+}(k-1)- \\
& -\sum_{j=0}^{l}\left(R(l-j, 0)+\sum_{i=1}^{k-l-1} n_{-}\left(n_{0}-l-1-i, i\right) R(l-j+i, 0)\right) n_{+}(j, k-1-j)+ \\
& +\sum_{j=l+1}^{k-2}\left(\sum_{0}^{-k-1} \sum_{i=0} n_{-}\left(i, n_{0}-l-1-i\right) R\left(n_{0}-j-1-i, 0\right)\right)^{t} n_{+}(j, k-1-j)
\end{aligned}
$$

(6.23) $\quad E_{n_{0}}(k-2 ; k)$

$$
\begin{aligned}
= & \sum_{i=0}^{n_{0}-k-1} n_{-}\left(i, n_{0}-k+1-i\right) R\left(n_{0}-k-i, 0\right)+n_{-}\left(n_{0}-k, 1\right) v_{+}(k-1)- \\
& -\sum_{j=0}^{k-2}\left(R(k-2-j, 0)+n_{-}\left(n_{0}-k, 1\right) R(k-1-j, 0)\right)^{t} n_{+}(j, k-1-j) \\
(6.24) \quad & E_{n_{0}}(k-1 ; k)=v_{+}(k-1)-\left(v+\sum_{j=0}^{k-2} R(k-1-j, 0) t n_{+}(j, k-1-j)\right) .
\end{aligned}
$$

By $(6.17)$,

(6.25)

$$
\begin{aligned}
c_{n_{0}}(k)=n_{+}\left(k-1, n_{0}-k+1\right) v_{+}(k-1)+\sum_{l=k}^{n_{0}-1} n_{+}\left(l, n_{0}-l\right) R(l+1-k, 0)+ \\
+\sum_{m=0}^{k-2}\left(\sum_{l=k}^{n_{0}-1} n_{+}\left(l, n_{0}-l\right) R(l-m, 0)\right)^{t} n_{+}(m, k-1-m) .
\end{aligned}
$$

Moreover, it follows from Theorem 3.1' and (iii) in step 5 that for any $m \in(0,1, \cdots, k-1)$,

(6.26)

$$
\mathrm{n}_{0}-1
$$

$$
\sum_{l=k} n_{+}\left(l, n_{0}-l\right) R(l-m, 0)
$$




$$
\begin{aligned}
& =\sum_{j=0}^{m-1} \delta_{+}\left(n_{0}-j\right)\left(\sum_{i=0}^{n_{0}-k-1} n_{-}\left(i, n_{0}-1-j-i\right) R\left(n_{0}-1-m-i, 0\right)\right)- \\
& -R\left(n_{0}-m, 0\right)-\sum_{j=0}^{k-1-m} n_{+}\left(j, n_{0}-m-j\right) R(j, 0) .
\end{aligned}
$$

By substituting (6.26) into (6.25), we find from (6.18) that

$$
\begin{aligned}
C_{n_{0}}(k)= & -D_{n_{0}}(k)+n_{+}\left(k-1, n_{0}-k+1\right) v_{+}(k-1)-\delta_{+}\left(n_{0}-k+1\right) v+ \\
& +\sum_{m=0}^{k-2}\left\{\sum_{j=0}^{m-1} \delta_{+}\left(n_{0}-j\right)\left(\sum_{i=0}^{n_{0}-k-1} n_{-}\left(i, n_{0}-1-j-i\right) R_{0}-1-m-1,0\right)\right)- \\
& \left.\quad-\sum_{j=0}^{k-1-m} n_{+}\left(j, n_{0}-m-j\right) R(j, 0)\right\} n_{+}(m, k-1-m)+ \\
& +\sum_{j=0}^{m-1} \delta_{+}\left(n_{0}-j\right)\left(\sum_{i=0} n_{-}\left(i, n_{0}-1-j-i\right) R\left(n_{0}-k-1,0\right)\right) .
\end{aligned}
$$

Further, it follows from Theorem $3.1^{\prime}$ that for any $m \in\{0,1, \cdots, k-2\}$,

$$
n_{+}\left(k-1, n_{0}-k+1\right)=\delta_{+}\left(n_{0}-k+1\right)+\sum_{j=1}^{k-1} \delta_{+}\left(n_{0}-k+1+j\right) n_{-}\left(n_{0}-k, j\right)
$$

$(6.29)$

$$
\begin{aligned}
& \sum_{j=0}^{k-1-m} n_{+}\left(j, n_{0}-m-j\right) R(j, 0) \\
& k-\sum_{j=0}^{1-m} \delta_{+}\left(n_{0}-m-j\right)\left(R(j, 0)+\sum_{i=j+1}^{k-1-m} n_{-}\left(n_{0}-m-i-1, j-j\right) R(i, 0)\right) .
\end{aligned}
$$

Thus, by substituting $(6.28)$ and $(6.29)$ into $(6.27)$, we can see that Step 9 holds.

(Q.E.D. )

$$
\text { (Step 10) } \quad E_{n_{0}}(k-1 ; k)=0 \text { for any } k \in\left(1, \cdots, n_{0}\right) \text {. }
$$

This follows from the assumption of mathematical induction that $(6.14)_{\mathrm{k}-1}$ holds.

(Step 11) $\quad E_{n_{0}}(k-2 ; k)=0$ for any $k \in\left\{2, \cdots, n_{0}\right\}$.

Proof. By (6.23) and $(6.14)_{k-1}$,

$$
\text { (6.30) } \begin{aligned}
E_{n_{0}}(k-2 ; k)= & \sum_{i=0}^{n_{0}-k-1} n_{-}\left(i, n_{0}-k+1-i\right) R\left(n_{0}-k-i, 0\right)+n_{-}\left(n_{0}-k, 1\right) v- \\
& -\sum_{j=0}^{k-2} R(k-2-j, 0){ }^{t} n_{+}(j, k-1-j) .
\end{aligned}
$$


By Theorem 3.1' and (i i i) in step 5 ,

$$
\begin{aligned}
& n_{0}-k-1 \\
& \sum_{i=0} n_{-}\left(i, n_{0}-k+1-i\right) R\left(n_{0}-k-i, 0\right) \\
= & -\delta_{-}\left(n_{0}-k+1\right) \delta_{+}\left(n_{0}-k\right) v+{ }_{0}^{n_{0}-k-3} \\
+ & \sum_{i=0} n_{-}\left(n_{0}-k\right)\left(R\left(n_{0}-k-1,0\right)+n_{0}^{-k-1}\right) R\left(n_{0}-k-2-i, 0\right)+ \\
& \left.\sum_{i=1} n_{+}\left(n_{0}-k-1-i, i\right) R\left(n_{0}-k-1-i, 0\right)\right) .
\end{aligned}
$$

By repeating the same procedure, we have

(6.31)

$$
\mathrm{n}_{0}-\mathrm{k}-1
$$

$$
\begin{aligned}
\sum_{i=0} n_{-}\left(1, n_{0}-k+1-i\right) R\left(n_{0}-k-i, 0\right) & =-\left(\sum_{i=1} \delta_{-}(i+1) \delta_{+}(i)\right) v \\
& =\left(-n_{-}\left(n_{0}-k, 1\right)+\delta_{-}(1)\right) v .
\end{aligned}
$$

On the other hand, by Theorem $3.1^{\prime}$,

$$
\begin{aligned}
& \sum_{j=0}^{k-2} R(k-2-j, 0)^{t} n_{+}(j, k-1-j) \\
= & \left(R(k-2,0)+v^{t} \delta_{-}(k-2)+\sum_{j=1}^{k-3} R(j, 0)^{t} n_{-}(j, k-2-j)\right)^{t} \delta_{+}(k-1)+ \\
& +\sum_{j=0}^{k-3} R(k-3-j, 0)^{t} n_{+}(j, k-2-j)
\end{aligned}
$$

and so by $(6.16) \mathrm{k}-2$

$$
\sum_{j=0}^{k-2} R(k-2-j, 0)^{t} n_{+}(j, k-1-j)=\sum_{j=0}^{k-3} R(k-3-j, 0)^{t} n_{+}(j, k-2-j) \text {. }
$$

By repeating the same procedure,

$$
\sum_{j=0}^{k-2} R(k-2-j, 0)^{t} n_{+}(j, k-1-j)=\delta_{-}(1) v \text {. }
$$

Thus, by (6.30), (6.31) and (6.32), we have Step 11 .

(Step 12) For any $\ell \in(0,1, \cdots, k-2)$.

$$
\begin{aligned}
E_{n_{0}}(l ; k)= & \sum_{j=0}^{n_{0}-k} n_{-}\left(i, n_{0}-l-i\right) R\left(n_{0}-k-i, 0\right)+ \\
& -\sum_{j=0}^{l}\left(R(l-j, 0)+\sum_{i=1}^{k-l-2} n_{-}\left(n_{0}-l-1-i, i\right) R(l-j+1,0)\right)^{t} n_{+}(j, k-1-j)+ \\
& -\sum_{j=l+1}^{k-2}\left(\sum_{i=j-l}^{k-l-2} n_{-}\left(n_{0}-l-1-i, i\right) R(i-j+l, 0)\right)^{t} n_{+}(j, k-1-j)+
\end{aligned}
$$




$$
+\sum_{j=l+1}^{k-2}\left(\sum_{i=j-l}^{n_{0}-l-1} n_{-}\left(n_{0}-\ell-1-i, i\right) R(i-j+l, 0)\right)^{t} n_{+}(j, k-1-j) .
$$

Proof. By substituting $(6.14)_{k-1}$ into (6.22), we have

(6.33)

$$
\begin{aligned}
& E_{n_{0}}(l ; k)=\sum_{i=0}^{n_{0}-k} n_{-}\left(i, n_{0}-\ell-1-i\right) R\left(n_{0}-k-i, 0\right)- \\
& -\sum_{j=0}^{\ell}\left(R(l-j, 0)+\sum_{i=1}^{k-l-2} n_{-}\left(n_{0}-l-1-i, i\right) R(l-j+1,0)\right)^{t} n_{+}(j, k-1-j)+ \\
& +\sum_{j=l+1}^{k-2}\left(\sum_{i=0}^{n} n_{0}\left(i, n_{0}-\ell-1-i\right) R\left(n_{0}-j-i-1,0\right)\right) t_{n}(j, k-1-j) .
\end{aligned}
$$

Since

$$
\begin{aligned}
& \sum_{i=j-l}^{k-l} n_{-}\left(n_{0}-l-1-i, i\right) R(i-j+l, 0)+\sum_{i=0}^{n_{0}-k} n_{-}\left(i, n_{0}-l-1-i\right) R\left(n_{0}-j-i-1,0\right) \\
= & \sum_{0}^{-l-1} \sum_{i=j-l} n_{-}\left(n_{0}-l-1-i, i\right) R(i-j+l, 0),
\end{aligned}
$$

it follows from (6.33) that step 12 holds.

(Q.E.D.)

For each $\ell, m \in \mathbb{N}$, put

$$
\text { (6.34) } \quad F(m, l)=\sum_{i=0}^{m} n_{-}(m-1, l+i) R(1,0) \text {. }
$$

(Step 13) (i) $F(m, 1)=\delta_{-}(1) V$

$$
\begin{array}{ll}
\text { (i i ) } & F(1, l)=\left(n_{-}(1, l)-\delta_{-}(l+1) \delta_{+}(1)\right) v \\
\text { (i i i ) } & F(m, l)=F(m-1, l)+\delta_{-}(m+l) \sum_{j=1}^{l-1} \delta_{+}(m+j) F(m-1, j) .
\end{array}
$$

Proof. (i) and (ii) follow from (6.31) and Step 2, respectively. By using Theorem 3.1' repeatedly,

$$
\begin{aligned}
F(m, l)= & F(m-1, \ell)+\delta_{-}(m+l)\left(\sum_{i=0}^{m-1} n_{+}(l+i-1, m-i) R(i, 0)+R(m, 0)\right) \\
= & F(m-1, l)+\delta_{-}(m+l) \sum_{j=1}^{l-1} \delta_{+}(m+j) F(m-1, j)+ \\
& +\delta_{-}(m+l)\left(\sum_{j=0}^{m-1} n_{+}(j, m-j) R(j, 0)+R(m, 0)\right),
\end{aligned}
$$


which, together with (i1i) in step 5 , implies (iii).

(Q.E.D.)

For each $\& \in(0, \cdots, k-2)$, put

$(6.35)$

$$
G(\ell ; k)=\sum_{i=0}^{l} R(l-i, 0)^{t} n_{+}(i, k-1-1) \text {. }
$$

(Step 14) For any $\& \in\{0, \cdots, k-2\}$,

$$
\begin{aligned}
E_{n_{0}}(l ; k)= & -G(l ; k)-\sum_{i=1}^{k-l-2} n_{-}\left(n_{0}-l-1-i, i\right) G(l+i ; k)+ \\
& +F\left(n_{0}-k, k-l-1\right)+\sum_{i=1}^{k-l-2} F\left(n_{0}-k+i, k-l-1-i\right) n_{+}(k-1-i, i) .
\end{aligned}
$$

This follows from Step $12,(6.34)$ and $(6.35)$.

( Step 15) For any $k \in\left(2, \cdots, n_{0}\right)$ and any $\ell \in\{0, \cdots, k-2\}$,

$$
E_{n_{0}}(l ; k)=E_{n_{0}-1}(l ; k)+\delta_{-}\left(n_{0}-l-1\right) \sum_{j=2}^{k-l-1} \delta_{+}\left(n_{0}-l-j\right) E_{n_{0}-1}(l-1+j ; k) \text {. }
$$

Proof. By (111) in Step 13,

$$
\begin{aligned}
& F\left(n_{0}-k, k-l-1\right)+\sum_{i=1}^{k-l-2} F\left(n_{0}-k+1, k-l-1-i\right)^{t} n_{+}(k-1-i, i) \\
& =F\left(n_{0}-k-1, k-l-1\right)+\delta_{-}(1) v^{t} n_{+}(l+1, k-l-2)+ \\
& +\sum_{i=1}^{k-l-3} F\left(n_{0}-k+1-1, k-l-1-i\right)^{t} n_{+}(k-1-1, i)+ \\
& +\delta_{-}\left(n_{0}-l-1\right) \sum_{j=2}^{k-l-2} \delta_{+}\left(n_{0}-l-j\right)\left(F\left(n_{0}-k-1, k-l-j\right)+\right. \\
& \left.+\sum_{k-l-1-j}^{k} F\left(n_{0}-k+i-1, k-l-j-i\right) n_{+}(k-1-i, i)\right)+ \\
& +\delta_{-}\left(n_{0}-l-1\right) \delta_{+}\left(n_{0}-k+1\right) F\left(n_{0}-k-1,1\right) .
\end{aligned}
$$

on the other hand, by Theorem 3.1'.

$$
\begin{aligned}
n_{-}\left(n_{0}-l-1-i, i\right)= & n_{-}\left(n_{0}-l-2-i, i\right)+\delta_{-}\left(n_{0}-l-1\right) \delta_{+}\left(n_{0}-l-1-i\right)+ \\
& +\delta_{-}\left(n_{0}-l-1\right) \sum_{j=1}^{1-1} \delta_{+}\left(n_{0}-l-1-j\right) n_{-}\left(n_{0}-l-2-i, j\right)
\end{aligned}
$$

and so

$(6.37)$

$$
\sum_{i=1}^{k-\ell-2} n_{-}\left(n_{0}-\ell-1-i, i\right) G(l+i ; k)
$$




$$
\begin{aligned}
= & \sum_{i=1}^{k-l-2} n_{-}\left(n_{0}-l-2-1, i\right) G(l+1 ; k)+\delta_{-}\left(n_{0}-l-1\right) \delta_{+}\left(n_{0}-k+1\right) G(k-2 ; k)+ \\
+ & \delta_{-}\left(n_{0}-l-1\right) \sum_{j=1}^{k-l-3} \delta_{+}\left(n_{0}-l-1-j\right)(G(l+j ; k)+ \\
& \left.+\sum_{i=j+1}^{k-l-2} n_{-}\left(n_{0}-l-2-i, j\right) G(l+i ; k)\right) .
\end{aligned}
$$

Hence, by combining step 14 with (6.36) and (6.37), we have step 15 .

(Q.E.D. )

(Step 16) For any $k \in\left\{2, \cdots, n_{0}\right\}$ and any $\ell \in(0, \cdots, k-2\}$,

$$
E_{n_{0}}(l ; k)=0 \text {. }
$$

Proof. By Step 15, it suffices to show that $E_{2}(0 ; 2)=0$, which is proved in step 11 .

(Q.E.D. )

(Step 17) For any $n \in \mathbb{N},(6.13)_{n}$ and $(6.14)_{n}$ hold.

This follows from step 8 Step 16.

Finally we will show ( 6.12 ) by a mathematical induction such that for any fixed $n_{0} \in\{2,3, \cdots\}$,

if

(6.38) $\quad R(j, k)=R(j-k, 0)$ for any $j, k \in \mathbb{N}^{*}, \quad 0 \leq k \leq j \leq n_{0}$,

then

(6.39) $\quad R\left(n_{0}+1, k\right)=R\left(n_{0}+1-k, 0\right)$ for any $k \in\left\{0, \cdots, n_{0}+1\right\}$.

(Step 18) For any $k \in\left(1, \cdots, n_{0}\right)$,

$R\left(n_{0}+1, k\right)=R\left(n_{0}, k-1\right)-\delta_{+}\left(n_{0}+1\right) H\left(k ; n_{0}\right)$,

where

(6.40) $\quad H(k ; n)=R(0 ; k)+\sum_{j=0}^{n-1} n_{-}(j, n-j) R(n-k, j) \quad(1 \leq k \leq n)$.

Proof. By multiplying both hand sides of (6.9) replaced $n$ by $n_{0}+1$ by ${ }^{t} x(k)$ and then taking an expectation with respect to $P$, $1 t$ follows from Step 1 and Theorem 3.1 ' that 


$$
\begin{aligned}
R\left(n_{0}+1, k\right)= & -\delta_{+}\left(n_{0}+1\right)\left(R(0, k)+\sum_{j=1}^{n_{0}} n_{-}\left(n_{0}-j, j\right) R(j, k)\right)- \\
& -\delta_{+}\left(n_{0}\right) R(1, k)-\sum_{0=1}^{n_{0}^{-1}} n_{+}\left(j, n_{0}-j\right) R(j+1, k) .
\end{aligned}
$$

And so by (6.11) and the assumption (6.38),

$$
\begin{aligned}
R\left(n_{0}+1, k\right)= & -\delta_{+}\left(n_{0}+1\right)\left(R(0, k)+\sum_{j=1}^{n_{0}} n_{-}\left(n_{0}-j, j\right) R\left(n_{0}-k, n_{0}-j\right)\right)- \\
& -\delta_{+}\left(n_{0}\right) R(0, k-1)-\sum_{j=1} n_{+}\left(j, n_{0}-j\right) R(j, k-1) \\
= & -\delta_{+}\left(n_{0}+1\right) H\left(k ; n_{0}\right)-\delta_{+}\left(n_{0}\right) R(0, k-1)- \\
& -n_{0}^{-1} \\
& \sum_{j=1} n_{+}(j, n-j) R(j, k-1) .
\end{aligned}
$$

which, together with (1) in step 4, yields Step 18.

(Q.E.D.)

$$
\text { ( Step 19) } \quad H(m ; m)=0 \text { for any } m \in \mathbb{N} \text {. }
$$

Proof. By Steps 6 and 17, we can apply (6.16) to get Step 19 .

(Q.E.D. )

(Step 20) For any $\ell \in\left(1, \cdots, n_{0}\right)$ and any $k \in(1, \cdots, \ell-1\}$,

$H(k ; \ell)=H(k ; \ell-1)+\delta_{-}(l) \sum_{j=1}^{k-1} \delta_{+}(\ell-j) H(k-j, l-1-j)$.

Proof. By the assumption (6.38),

$$
\begin{aligned}
H(k ; l) & =R(0, k)+\sum_{j=0}^{\ell-k} n_{-}(j, l-j) R(l-k, j)+\sum_{j=l-k+1}^{\ell-1} n_{-}(j, l-j) R(l-k, j) \\
& =R(0, k)+\sum_{l=0}^{\ell-k} n_{-}(j, l-j) R(l-k-j, 0)+\sum_{j=l-k+1}^{\ell} n_{-}(j, l-j) R(0, j-l+k) .
\end{aligned}
$$

By Theorem 3.1',

$$
\begin{aligned}
H(k ; \ell)= & R(0, k)+\sum_{j=0}^{\ell-k-1} n_{-}(j, l-j-1) R(l-1-j, 0)+ \\
& +\sum_{j=l-k}^{\ell-2} n_{-}(j, l-1-j) R(0, j-l+1+k)+ \\
& +\delta_{-}(l)\left(R(l-k, 0)+\sum_{j=1}^{\ell-k} n_{+}(l-j-1, j) R(l-k-j, 0)+\right.
\end{aligned}
$$




$$
\begin{gathered}
\left.+\sum_{j=l-k+1}^{\ell-1} n_{+}(\ell-j-1, j) R(0, j-l+k)\right) \\
=H(k ; \ell-1)+\delta_{-}(\ell)\left(R(l-k, 0)+\sum_{j=1}^{\ell-k} n_{+}(l-j-1, j) R(l-k-j, 0)+\right. \\
\left.\quad \sum_{j=l-k+1}^{\ell-1} n_{+}(l-j-1, j) R(0, j-l+k)\right) .
\end{gathered}
$$

By using Theorem 3.1' again,

$$
\begin{aligned}
H(k ; l)= & H(k ; l-1)+\delta_{-}(l) \delta_{+}(l-1) H(k-1 ; l-1)+ \\
& +\delta_{-}(\ell)\left(R(l-k, 0)+\sum_{j=1}^{\ell-k} n_{+}(l-j-2, j) R(l-k-j, 0)+\right. \\
& \left.+\sum_{j=\ell-k+1}^{\ell-2} n_{+}(l-j-2, j) R(0, j-l+k)\right) .
\end{aligned}
$$

The same repetition yields that

$$
\begin{aligned}
H(k ; l)= & H(k, l-1)+\delta_{-}(l) \sum_{j=1}^{k-1} \delta_{+}(l-j) H(k-j ; l-1-j)+ \\
& +\delta_{-}(l)\left(R(l-k, 0)+\sum_{j=1}^{\ell-k} n_{+}(l-k-j, j) R(l-k-j, 0)\right),
\end{aligned}
$$

which, together with ( $11 i$ ) in Step 5 , implies Step 20 . (Q.E.D.)

( Step 21) $R\left(n_{0}+1, k\right)=R\left(n_{0}, k-1\right)$ for any $k \in\left(1, \cdots, n_{0}\right)$.

Proof. By Steps 2 and 19 , we can apply a mathematical induction to see that $H(k ; l)=0$ for any $\ell \in\left\{1, \cdots, n_{0}\right\}$ and any $k \in$ $(1, \cdots, \ell)$. Hence Step 21 follows from Step 18 . (Q.E.D.)

( Step 22) $R\left(n_{0}+1, n_{0}+1\right)=R(0,0)$.

Proof. By multiplying both hand sides of (6.9) replaced $n$ by $n_{0}+1$ by ${ }^{t} X\left(n_{0}+1\right)$ and then taking an expectation with respect to $P$, it follows from $S$ tep 1 and the assumption (6.38) that

$$
\begin{aligned}
R\left(n_{0}+1, n_{0}+1\right)= & -\delta_{+}\left(n_{0}+1\right) R\left(0, n_{0}+1\right)+v_{+}\left(n_{0}+1\right)+ \\
& +\sum_{j=1} n_{+}\left(j, n_{0}+1-j\right) R\left(0, n_{0}+1-j\right) .
\end{aligned}
$$

By Steps 6 and 17 , we can substitute $(6.16)_{n_{0}+1}$ into the above to see that 


$$
\begin{aligned}
R\left(n_{0}+1, n_{0}+1\right)= & v_{+}\left(n_{0}+1\right)+\delta_{+}\left(n_{0}+1\right) \delta_{-}\left(n_{0}+1\right) v_{+}\left(n_{0}\right)- \\
& -\sum_{j=1}\left(n_{+}\left(j, n_{0}+1-j\right)-\delta_{+}\left(n_{0}+1\right) n_{-}\left(n_{0}-j, j\right)\right) R\left(0, n_{0}+1-j\right)
\end{aligned}
$$

and so by Theorems $3.1^{\prime}$ and $4.1(11)$,

$$
\begin{aligned}
R\left(n_{0}+1, n_{0}+1\right) & =v_{+}\left(n_{0}\right)-\sum_{j=1}^{n_{0}} n_{+}\left(j-1, n_{0}+1-j\right) R\left(0, n_{0}+1-j\right) \\
& =v_{+}\left(n_{0}\right)-\sum_{j=1}^{n_{0}} n_{+}\left(n_{0}-j, j\right) R(0, j) .
\end{aligned}
$$

Therefore, by Step 17, we can apply (6.14) $n_{0}+1$ to the above to see that $R\left(n_{0}+1, n_{0}+1\right)=R(0,0)$.

(Q.E.D.)

Thus, we have completed the proof of Theorem 6.1.

Added in proof After thls paper was prepared, the author learned from Doctor of Engineering H. Sakal that the algorithm (1), (ii) in Theorem 3.1, (ii), (iv) in Theorem 4.1 and (i), (ii) in Theorem 4.2 has been derived for the fitting of a multi-dimensional autoregressive model [N. Levinson, J. Math. Phys, 25(1947), 261-278; J . Durbin, Biometrika, 46(1959), 306-316; P. Whittle, Biometrika, 50 (1963), 129-134; R. A. Wiggins and E. A. Robinson, J. Geophys. Res., 70(1965), 1885-1891]. This algorithm is called Levinson-Durbin algorithm for one-dimensional case and Levinson-Whittle-Wiggins-Robinson algorithm for multi-dimensional case. In particular, the fundamental relation in Lemma 4.3 , and parameters $\delta_{+}(\cdot)$ and $\delta_{-}(\cdot)$ are called Burg's relation and partial autocorrelation coefficients, respectively. The role that autoregressive processes have in our theory of $\mathrm{KM}_{2} \mathrm{O}^{-}$ Langevin equations will be discussed in [27]. The author would like to thank Dr. Prof. H. Sakai for his communication. 


\section{References}

[1] L. de Branges : Hilbert Spaces of Entire Functions, PrenticeHall, Englewood Cliffs, New Jersey, 1968.

[2] H. Dym and H. P. McKean, Jr.: Application of de Branges spaces of integral functions to the prediction of stationary Gaussian processes, Illinois J. Math. 45(1970), 299-343.

[3] H. Dym and H. P. McKean, Jr. : Gaussian processes, Function Theory, and the Inverse spectral problem, Academic Press, New York, 1976.

[4] A. N. Kolmogorov: Interpolation und extrapolation von stationaren zufälligen folgen, Dokl. Adad. Nauk SSSR, $5(1941), 3-14$.

[5] M. G. Krein: On a fundamental approximation problem in the theory of extrapolation and filtration of stationary random processes, Dokl. Akad. Nauk SSSR, 94(1954), 13-16.

[6] R. Kubo: Statistical mechanical theory of irreversible processes 1 , general theory and simple applications to magnetic and conduction problems, J. Phys. Soc. Japan, $12(1957), 570-586$.

[7] T. Miyoshi : On $(\ell, m)$-string and $(\alpha, \beta, \gamma, \delta)$-Langevin equation assoceated with a stationary Gaussian process, J. Fac. Sci. Univ. Tokyo, Sect. IA,30(1983), 139-190.

[8] T. Miyoshi: On an $\mathbb{R}^{\mathrm{d}}$-valued stationary Gaussian process associated with $(k, l, m)-s$ tring and $(\alpha, \beta, \gamma, \delta)$-Langevin equation, J. Fac. Sci. Univ. Tokyo, Sect.IA,31(1984), 155-194.

[9] H. Mori : Transport, collective motion and Brownian motion, Progr. Theor. Phys. 33(1965), 115-165.

[10] Y. Nakano and Y. Okabe : On a multi-dimensional $[\alpha, \beta, \gamma]-$ Langevin equation, Proc. Japan Acad., 59(1983), 171-173.

[11] Y. Okabe : Stationary Gaussian processes with Markovian property and M. Sato's hyperfunctions, Jap. J. Math., 41(1973), 69-122.

[12] Y. Okabe: On a stationary Gaussian process with T-positivity and its associated Langevin equation and $S-m a t r i x, J$. Fac. Sci. Univ. Tokyo, Sect. IA, 26(1979), 115-165.

[13] Y. Okabe: On a stochastic differential equation for a stationary Gaussian process with T-positivity and the fluctuation-dissipation theorem, J. Fac. Sci. Univ. Tokyo, Sect.IA, 28(1981), 169-213.

[14] Y. Okabe: On a stochastic differential equation for a 
stationary Gaussian process with finite multiple Markovian property and the fluctuation-dissipation theorem, J. Fac. Sci. Univ. Tokyo, Sect.IA, 28(1982), 793-804.

[15] Y. Okabe : On a Langevin equation, Sugaku, 33(1981), 306-324 (in Japanese).

[16] Y. Okabe : On a wave equation associated with predlction errors for a stationary Gaussian process, Lecture Notes in Control and Information Sclences, 49(1983), 215-226.

[17] Y. Okabe : A generalized fluctuation-dissipation theorem for the one-dimensional diffusion process, Commun. Math. Phys., $98(1985), 449-468$.

[18] Y. Okabe : On KMO-Langevin equations for stationary Gaussian process with T-positivity, J. Fac. Sci. Univ. Tokyo, Sect.IA, $33(1986), 1-56$.

[19] Y. Okabe : On the theory of Brownian motion with the AlderWainwright effect, J. Stat. Phys., 45(1986), 953-981.

[20] Y. Okabe : KMO-Langevin equation and fluctuation-dissipation theorem (I), Hokkaido Math. J., 15(1986), 163-216.

[21] Y. Okabe : KMO-Langevin equation and fluctuation-dissipation theorem (II), Hokkaido Math. J., 15(1986), 317-355.

[22] Y. Okabe : Stokes-Boussinesq-Langevin equation and fluctuationdissipation theorem, Prob. Theory and Math. Stat., vol.2, 431-436, Prohorov et al.(eds), 1986 VNU Science Press.

[23] Y. Okabe: On long time talls of correlation functions for KMO-Langevin equations, to appear in Proceeding of Fourth Japan-USSR symposium on probability theory, Kyoto, July, 1986.

[24] Y. Okabe: On the theory of discrete KMO-Langevin equations with reflection positivity (I), to appear in Hokkaido Math. J.

[25] Y. Okabe: On the theory of discrete KMO-Langevin equations with reflection positivity (II), to be submitted to Hokkaido Math. J.

[26] Y. Okabe: On the theory of discrete KMO-Langevin equations with reflection positivity (III), in preparation.

[27] Y. Okabe: On stochastic difference equations for the multidimensional weakly stationary time series, in preparation.

[28] N. Wiener : Extrapolation, interpolation, and smoothing of stationary time series, Wiley, New York, 1949. 